\title{
G

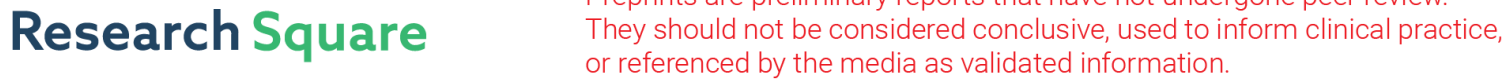 \\ Clonally Expanded B Cells in Multiple Sclerosis Bind EBV EBNA1 and GlialCAM
}

\section{Tobias V Lanz}

Stanford School of Medicine https://orcid.org/0000-0001-7106-8801

R Camille Brewer

Stanford School of Medicine https://orcid.org/0000-0002-0342-1826

Peggy P Ho

Stanford School of Medicine https://orcid.org/0000-0002-6848-318X

\section{Kevin M Jude}

Stanford School of Medicine https://orcid.org/0000-0002-3675-5136

\section{Daniel Fernandez}

Stanford School of Medicine https://orcid.org/0000-0002-6221-152X

Jae-Seung Moon

Stanford School of Medicine https://orcid.org/0000-0002-1983-465X

\section{Ricardo A Fernandes}

Stanford School of Medicine https://orcid.org/0000-0001-5343-3334

\section{Alejandro M Gomez}

Stanford School of Medicine

\section{Gabriel-Stefan Nadj}

Stanford School of Medicine https://orcid.org/0000-0002-9938-431X

\section{Christopher M Bartley}

University of California San Francisco https://orcid.org/0000-0002-1391-4130

\section{Ryan D Schubert}

University of California San Francisco https://orcid.org/0000-0002-3132-5831

Isobel A Hawes

University of California San Francisco https://orcid.org/0000-0003-1719-0114

\section{Sara E Vazquez}

University of California San Francisco

Manasi lyer

Stanford School of Medicine

\section{J Bradley Zuchero}

Stanford School of Medicine https://orcid.org/0000-0002-4706-2538

\section{Bianca Teegen}


Euroimmun

Jeffrey E Dunn

Stanford School of Medicine

Christopher B Lock

Stanford School of Medicine

Lucas B Kipp

Stanford School of Medicine

Victoria C Cotham

NYU School of Medicine

\section{Beatrix M Ueberheide}

NYU School of Medicine https://orcid.org/0000-0003-2512-0204

\section{Blake T Aftab}

Atara Bio https://orcid.org/0000-0003-4020-2718

\section{Mark S Anderson}

University of California San Francisco

Joseph L DeRisi

University of California San Francisco https://orcid.org/0000-0002-4611-9205

\section{Michael R Wilson}

University of California San Francisco https://orcid.org/0000-0002-8705-5084

\section{Rachael JM Bashford-Rogers}

Wellcome Centre for Human Genetics, University of Oxford https://orcid.org/0000-0002-6838-0711

\section{Michael Platten}

Medical Faculty Mannheim, University of Heidelberg https://orcid.org/0000-0002-4746-887X

\section{K Christopher Garcia}

Stanford School of Medicine

\section{Lawrence Steinman}

Stanford School of Medicine https://orcid.org/0000-0002-2437-2250

William H Robinson ( $\sim$ wrobins@stanford.edu )

Stanford School of Medicine https://orcid.org/0000-0003-4385-704X

\section{Research Article}

Keywords: Epstein-Barr Virus, EBV, multiple sclerosis, MS, antibodies, molecular mimicry, inflammation, B cells, Immune Repertoire

Posted Date: January 11th, 2022

DOI: https://doi.org/10.21203/rs.3.rs-1239863/v1 
License: (c) (i) This work is licensed under a Creative Commons Attribution 4.0 International License. Read Full License

Version of Record: A version of this preprint was published at Nature on January 24th, 2022. See the published version at https://doi.org/10.1038/s41586-022-04432-7. 


\section{Abstract}

Multiple sclerosis (MS) is a heterogenous autoimmune disease in which autoreactive lymphocytes attack the myelin sheath of the central nervous system (CNS). B lymphocytes in the cerebrospinal fluid (CSF) of MS patients contribute to inflammation and secrete oligoclonal immunoglobulins. Epstein-Barr virus (EBV) infection has been linked to MS epidemiologically, but its pathological role remains unclear. Here we demonstrate high-affinity molecular mimicry between the EBV transcription factor EBNA1 and the CNS protein GlialCAM, and provide structural and in-vivo functional evidence for its relevance. A cross-reactive CSF-derived antibody was initially identified by single-cell sequencing of the paired-chain B cell repertoire of MS blood and CSF, followed by protein microarray-based testing of recombinantly expressed CSFderived antibodies against MS-associated viruses. Sequence analysis, affinity measurements, and the crystal structure of the EBNA1-peptide epitope in complex with the autoreactive Fab fragment allowed for tracking the development of the naïve EBNA1-restricted antibody to a mature EBNA1/GlialCAM crossreactive antibody. Molecular mimicry is facilitated by a post-translational modification of GlialCAM. EBNA1 immunization exacerbates the mouse model of MS and anti-EBNA1/GlialCAM antibodies are prevalent in MS patients. Our results provide a mechanistic link for the association between MS and EBV, and could guide the development of novel MS therapies.

\section{Main Text}

The presence of oligoclonal bands (OCB) in cerebrospinal fluid (CSF) and the efficacy of B cell depleting therapies emphasize the importance of $B$ cells in the pathobiology of multiple sclerosis (MS) ${ }^{2}$. Anti-viral antibodies against mumps, measles, varicella-zoster, and Epstein-Barr Virus (EBV) are often present in

$\mathrm{MS}^{4,5}$, but their relevance is unclear. Anti-EBV antibody titers in over $99 \%$ of MS patients provide evidence for an epidemiological link between MS and EBV 6 . Symptomatic infectious mononucleosis during EBV infection increases risk for $\mathrm{MS}^{7}$. Molecular mimicry between virus and self-antigens is a potential mechanism that might explain this association ${ }^{8}$. Antibodies against certain EBV nuclear antigen 1 (EBNA1) regions have been found in MS patients, including the region AA365-4265,9-12, which we describe here in our identification of molecular mimicry between EBNA1 and the glial cellular adhesion molecule GliaICAM. The potential significance of this mimicry in the pathophysiology of MS is described in detail.

\section{The B cell repertoire in MS CSF plasmablasts is highly clonal}

CSF and blood samples were obtained from MS patients during the onset of disease (clinically isolated syndrome, $n=5)$ or an acute episode of relapsing-remitting MS ( $n=4)$. Patients with a CSF pleocytosis of $>10$ cells/ $\mu$ l were selected (Extended Data Table 1, Supplementary Discussion). Single B cells were sorted by flow cytometry (Extended Data Fig. 1a,b). Characteristic phenotypic differences of B cells in blood and CSF were observed ${ }^{13,14}$, including (i) high plasmablast (PB) counts in CSF vs. blood (Extended Data Fig. 
1c,d), (ii) differing expression levels of integrin-a4 and HLA-DR in PB but not non-PB B cells (Extended Data Fig. 1e-j, Supplementary Table 1), and (iii) high abundance of immunoglobulin G (IgG) in CSF PB (Fig. 1a, Extended Data Fig. 1k,l).

We sorted PB from blood and B cells from matched CSF by flow cytometry, and sequenced their fulllength paired heavy-chain (HC) and light-chain (LC) VDJ regions ${ }^{15} .13,578$ paired sequences from blood PB and 1,689 from CSF B cells passed filter thresholds. The CSF repertoire is highly clonal (Fig. 1a, Extended Data Fig. 2a,3), suggesting antigen-specific proliferation of select clones within the CSF. Notably, CSF repertoires in three non-MS control patients with neuroinflammatory conditions (Extended Data Table 1) did not exhibit such extensive clonality and high IgG usage (Fig. 1a,b, Extended Data Fig. 2b-d). While the amount of somatic hypermutation (SHM) in immunoglobulin HC-V genes (IGHV) and LC$\checkmark$ genes (IGLV) does not differ between PB in blood and CSF (Extended Data Fig. 2e), HCcomplementarity determining region 3 (CDR3) lengths are longer in CSF PB than in blood, suggesting ongoing intrathecal SHM (Extended Data Fig. 2f). Consistent with previous reports ${ }^{16-19}$, repertoires in the CSF are skewed towards usage of five IGHV genes (Fig. 1c, Extended Data Fig. 2g-i). This suggests that select autoantigens drive PB survival and proliferation in CSF.

\section{Clonal PB are the predominant source of oligoclonal immunoglobulins in MS CSF}

Immunoglobulin levels are elevated in CSF of MS patients, and the presence of a few highly abundant OCB is a hallmark of MS. To determine if clonal PB are the source of intrathecal immunoglobulins, we isolated immunoglobulins from CSF, characterized them by mass spectrometry, and compared the spectra with their corresponding antibody repertoire sequence datasets. For $87 \%$ of clonal sequences, peptides matching variable chain sequences unique to the patient were identified, whereas this occurred only for $40 \%$ of singleton sequences (Fig. 1d). Highly abundant immunoglobulins, defined by detection of $\geq$ ten peptide-spectral matches ( $P S M \geq 10$ ), aligned almost exclusively to clonally expanded $B$ cells (Extended Data Fig. 2j) and to PB, which display more clonality than non-PB B cells (Extended Data Fig. $2 \mathrm{k}, \mathrm{l})$, suggesting that clonally expanded $\mathrm{PB}$ are the predominant source of CSF OCB.

\section{CSF-derived monoclonal antibodies bind EBV antigens}

A total of 148 BCR sequences from MS CSF, each representative of a clonal expansion, were expressed as monoclonal antibodies (mAbs) (Extended Data Fig. 3). MS CSF mAbs showed low polyreactivity (polyreactive mAbs MS: 5.4\%; controls: 15.4\%; Extended Data Fig. 4, 5b). To test for anti-viral reactivities, mAbs were probed on protein microarrays containing 2 EBV viral lysates, 23 latent and lytic EBV proteins, 240 peptides spanning four prominent EBV proteins, and lysates of 7 other MS-associated viruses, including measles, rubella, and varicella-zoster virus (VZV) (Fig. 2a,b, Extended Data Fig. 5a, 6, 7a, Supplementary Tables 2,3). One-third of the expressed mAbs bind to EBV proteins and peptides and 
$\sim 20 \%$ to other viruses, in particular to VZV and cytomegalovirus (CMV) (Fig. 2a). Notably, half of the VZVreactive antibodies cross-react to $\mathrm{CMV}$ and $\mathrm{EBV}$, indicative of conserved epitopes in herpes viruses.

Interestingly, we found mAbs from 6 out of 9 MS patients that bind to the EBV transcription factor EBNA1 (Fig. 2a, Extended Data Fig. 5a), and mAbs binding to EBNA1 peptides were identified in 8 of 9 patients (Fig. 2b, Extended Data Fig. 6). Anti-EBNA1-reactivity has been implicated in MS epidemiology ${ }^{20}$, and the

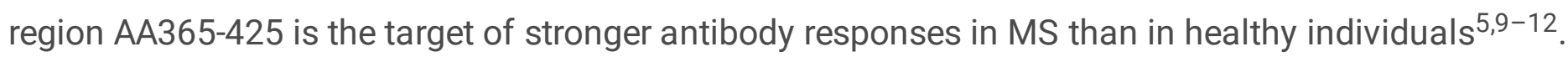
Protein and peptide microarrays revealed that mAb MS39p2w174 binds EBNA1 within this MS-associated region at AA386-405 (Fig. 2b, Extended Data Fig. 6). This epitope was confirmed by western blot analysis using full-length and truncated EBNA1 proteins (Fig. 2c), and by ELISA-based EBNA1 peptide scans (Fig. 2d). Alanine scanning identified the proline/arginine-rich region AA394-399 as the central epitope for MS39p2w174 (Fig. 2e, Extended Data Fig. 7b).

\section{Crystal structure reveals key residues of MS39p2w174-Fab / EBNA1 AA386-405 $_{\text {interaction }}$}

While the presence of antibodies against the broader EBNA1 region AA365-425 has been described in $\mathrm{MS}^{5,9-12}$, their relevance remains elusive. We solved the crystal structure of EBNA1 ${ }_{A A 386-405}$ in complex with the MS39p2w174-Fab at a resolution of $2.5 \AA$ (Fig. 2f-j, Extended Data Fig. 7c-e, PDB ID: 7K7R ${ }^{21}$ ). We demonstrate close interactions of the EBNA1 residues P394-P398 with all CDRs except for LC-CDR2. Residues Tyr31 and Tyr38 on LC-CDR1 together with Trp38 on HC-CDR1 and Pro108, Pro109, and Tyr114 on HC-CDR3 create a hydrophobic cage for the peptide's two N-terminal prolines Pro394 and Pro395 and the proximal side chain of Arg396 (Fig. 2h,i). The C-terminal end of the antibody binding groove is wider, and Pro398 is carried by a large aromatic tryptophan residue (Trp114 in HC-CDR1)(Fig. 2g,h). The central arginines Arg395 and Arg396 hydrogen bond with residues on HC-CDR2, HC-CDR3, and HC framework region 2.

The IGHV encoding gene of MS39p2w174 is IGHV3-7, one of the IGHV chains over-represented in CSF (Fig. 1c). Interestingly, all but two of the residues that directly interact with EBNA1 are unmutated germline residues (Extended Data Fig. 7f,g). We hypothesized that the unmutated ancestor of MS39p2w174 (germline) might have an innate propensity to bind EBNA1 AA386-405. Indeed, germline was significantly more polyreactive than MS39p2w174 (Extended Data Fig. 4), and bio-layer interferometry revealed that germline binds EBNA1 with only slightly lower affinity than MS39p2w174 (K MS39p2w174: $1.99 \mathrm{nM}, \mathrm{SD}: 0.63 \mathrm{nM}$; germline: $4.19 \mathrm{nM}, \mathrm{SD}: 0.76 \mathrm{nM}$ ) (Fig. 2k,l), indicating that SHM is not required for effective EBNA1 binding and that naïve B cells have EBNA1-specificity.

\section{Molecular mimicry between EBNA1 ${ }_{\mathrm{AA} 386-405}$ and GlialCAM}


We probed mAb MS39p2w174 on HuProt microarrays, which contained $>16,000$ proteins spanning $>80 \%$ of the human proteome ${ }^{22}$, and discovered that MS39p2w174 binds glial cell adhesion molecule (GlialCAM) (Fig. 3a). GlialCAM is an immunoglobulin superfamily cell adhesion molecule expressed in

the CNS (Extended Data Fig. 8a, www.proteinatlas.org ${ }^{23}$ ) by astrocytes and oligodendrocytes ${ }^{24-26}$. Earlier proteomics studies on MS brain lesions by our laboratory revealed expression of GlialCAM in chronicactive plaques ${ }^{27}$. MS39p2w174 was also probed using human proteome-wide phage display immunoprecipitation and sequencing (PhIP-Seq) library ${ }^{28}$. MS39p2w174 did not highly enrich for any single peptide, suggesting low affinity for multiple native peptides (Extended Data Table 2). Peptide motif analysis identified a common Pro/Arg-rich motif that closely resembles the central epitope in EBNA1 (AA395-399, Fig. 2e, Extended Data Fig. 8b). Comparing PhIP-Seq results with Huprot results yielded two overlapping targets: the ubiquitously expressed actin filament associated protein 1 (AFAP1, without the Pro/Arg-rich motif) and the CNS protein GliaICAM (=HEPACAM). Binding of MS39p2w174 to the intracellular domain (ICD, AA262-416) of GlialCAM was confirmed by ELISA (Fig. 3b) and western blot (Fig. 3c). Several regions in mouse brain distinctly stain for MS39p2w174 with predominantly glial patterns, including radial Bergmann glia in cerebellum, glia limitans, and likely perivascular glial cells in the hippocampus and brainstem (Fig. 3d, Extended Data Fig. 8c). MS39p2w174 stained rat oligodendrocytes and transgenic GlialCAM expressing K562 cells, where GlialCAM generates tight junctions (Extended Data Fig. 8d-f).

While we demonstrated that MS39p2w174 and its unmutated germline ancestor bound EBNA1 with similar affinity (Fig. 2k,l), their binding affinities to GlialCAM differ significantly: affinity of MS39p2w174 to GlialCAM is $\geq 10$-fold increased ( $K_{D}$ EBNA1: 1.99 nM, SD: 0.63 nM; vs. $K_{D}$ GlialCAM: 190 pM, SD: $17 \mathrm{pM}$ ), while germline binds GlialCAM with lower affinity ( $\mathrm{K}_{\mathrm{D}}$ EBNA1: $4.19 \mathrm{nM}, \mathrm{SD}: 0.76 \mathrm{nM}$ vs. $\mathrm{K}_{\mathrm{D}}$ GlialCAM: 10.46 nM, SD: 4.12 nM) (Fig. 3e,f). Evidently, while germline harbors a propensity to bind to EBNA1, SHM of MS39p2w174 increased its affinity to the CNS mimic GlialCAM by an order of magnitude.

\section{Phosphorylation of GlialCAM ${ }_{\text {AA370-389 }}$ at pSer376 enables MS CSF-derived MS39p2w174 binding}

The EBNA1 epitope AA386-405 is located between the protein's long N-terminal Gly-Ala-rich lowcomplexity region (AA90-380) and its highly structured DNA-binding domain (AA461-607, PDB: 1B3T'29). The GlialCAM region AA337-385, identified by phage display (Supplementary Table 4), is located at the Cterminal end of the ICD and contains a proline-rich region that resembles the central epitope of EBNA1 (Fig. 3g). Intracellular B cell autoantigens have been described in several autoimmune diseases ${ }^{30-32}$. MS39p2w174 reacts with both proteins on western blots under denaturing conditions (Fig. 2c, Fig. 3c), suggesting linear epitopes for both targets. This is consistent with predictions that both epitopes are located in intrinsically disordered regions of their respective proteins (Fig 3h,i). 
Nevertheless, while MS39p2w174 binds with similar affinity to both the EBNA1 protein and EBNA1 ${ }_{A A 386-}$ 405 peptide ( $K_{D}$ protein: $1.99 \mathrm{nM}, S D: 0.63 \mathrm{nM} ; K_{D}$ peptide: $2.67 \mathrm{nM}, \mathrm{SD}: 0.078 \mathrm{nM}$ ), its binding affinity for the native peptide GlialCAM ${ }_{A A 370-389}$ is three orders of magnitude lower than for GlialCAM protein $\left(K_{D}\right.$ protein: 190 pM, SD: 17pM; K $\mathrm{K}_{\mathrm{D}}$ peptide: $302 \mathrm{nM}, \mathrm{SD}: 31 \mathrm{nM}$ ). Part of this discrepancy could be due to multimerization of GlialCAM ${ }^{33}$. Additionally, the intracellular domain of GlialCAM is heavily phosphorylated ${ }^{33-35}$ (Extended Data Fig. 8g, phosphosite.org ${ }^{36}$ ), and post-translational modifications (PTMs) often determine antibody-antigen interactions ${ }^{37}$. We determined if phosphorylation at one of the four serine residues surrounding the central epitope region (residues Ser376, 377, 383, and 384) could

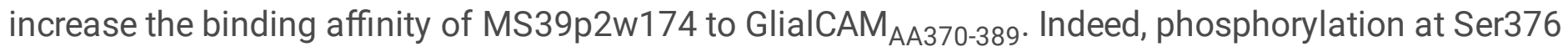
increased MS39p2w174 binding by 50-fold ( $K_{D}$ native peptide: $302 \mathrm{nM}, \mathrm{SD}: 0.078 \mathrm{nM} ; \mathrm{K}_{\mathrm{D}}$ pSer376 peptide: $6.1 \mathrm{nM}, \mathrm{SD}: 0.27 \mathrm{nM}$ ), and additional phosphorylation of Ser377 further enhanced binding affinity ( $K_{D}$ pSer376/pSer377 peptide: 3.73 nM, SD: $0.15 \mathrm{nM}$ ) (Fig. 3j-I). In contrast, citrullination of arginine residues Arg373, 380, and 387 did not alter binding to GlialCAM ${ }_{\text {AA370-389 }}$ (Extended Data Fig. 8h). The important residue Arg397 in EBNA1 ${ }_{A A 386-405}$, which engages in two hydrogen-bonds with Glu64 at

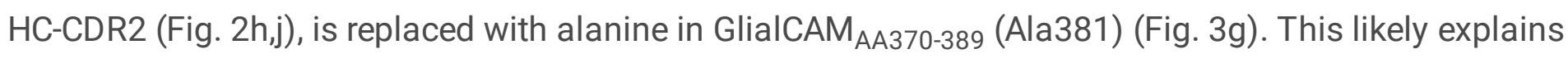
the differential binding affinity to EBNA1 and GlialCAM peptides. Phosphorylation at Ser376 likely promotes binding by providing new polar interactions to the proximal LC, possibly with Arg36, a positively charged residue that is mutated from asparagine in germline (Fig. 2h, Extended Data Fig. 7g). Together, our results show that post-translational phosphorylation enables cross-reactivity of anti-EBNA1 MS39p2w174 to GlialCAM.

\section{Anti-GlialCAM IgG reactivity is elevated in MS patients}

To determine if the observed anti-GlialCAM reactivity of MS39p2w174 represents a broader phenomenon in MS, we tested the remaining 147 clonal MS mAbs for reactivity against GlialCAM protein and peptides spanning GlialCAM $\mathrm{AA315-395}$. We found ten additional mAbs from seven patients that bound the ICD and seven more from four patients that bound the extracellular domain (ECD) (Fig. 3m), demonstrating that MS PBs generate antibodies against multiple GlialCAM epitopes. We then tested plasma samples from MS patients $(n=36)$ and healthy individuals $(n=20)$ for reactivity to EBNA1 and GlialCAM. As expected, all MS patients and the majority of healthy individuals exhibited plasma reactivity to EBNA1 protein (Fig. 3n). Reactivity to EBNA1 $1_{A A 386-405}$ and to GlialCAM was significantly increased in MS patients (Fig. 3o,p). Increased plasma IgG levels against EBNA1 ${ }_{A A 386-405}$, GlialCAM ${ }_{A A 370-389}$, and phosphorylated

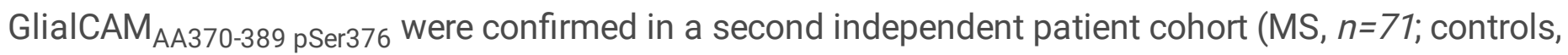
$n=50$; Extended Data Fig. 9a). Similarly, we detected increased plasma IgG levels against GlialCAM, GlialCAM $_{\text {AA370-389, }}$ and phosphorylated GlialCAM ${ }_{\text {AA370-389 pSer376 }}$ in a third cohort (MS, $n=67$; controls, $n=31$; Extended Data Fig. 9b). To address if reactivity against EBNA1 $1_{A A 386-405}$ could be blocked by GlialCAM, we showed inhibition of anti-EBNA1 ${ }_{\text {AA386-405 }}$ reactivity by GlialCAM AA370-389 pSer376 $_{\text {in nine }}$ 
high-reactivity samples (Fig. 3q, Extended Data Fig. 9c), indicating that this molecular mimicry is prevalent in a subset of MS patients.

\section{Immunization with EBNA1 ${ }_{\text {AA386-405 }}$ aggravates $\mathrm{EAE}$}

To assess the effect of an anti-EBNA $1_{A A 386-405}$ immune response on neuroinflammation, we utilized experimental autoimmune encephalomyelitis (EAE), the mouse model of MS. SJL/J mice were immunized with scrambled control peptide or EBNA1 $A A 386-405$. Three weeks later, EAE was induced by a second immunization of the respective peptides mixed with PLP $_{\text {AA139-151. Mice in the EBNA1 }}$ AA386-405 group generated robust antibody responses to both EBNA1 AA386-405 (Fig. 4a) and GlialCAM ICD (Fig. 4b). The antibody response to PLP AA139-151 was unaltered in both groups (Extended Data Fig. 10a). In addition, EBNA1 ${ }_{A A 386-405}$ immunization induced a strong $C D 4+T$ cell response while the $T$ cell response against PLP $\mathrm{AA} 139-151_{15}$ remained stable in both groups (Extended data Fig. 10b). EBNA1 AA386-405 stimulated the secretion of B cell stimulatory Th1 cytokines IFN- $\gamma$, TNF, and IL-12, as well as IL-6 and IL-10 (Extended Data Fig. 10c-g), but suppressed the key Th17 cytokine IL-17 (Extended Data Fig. 10h). Clinically, the EBNA1 group exhibited more severe paresis (Fig. 4c), with enhanced CNS immune cell infiltration (Fig. 4d,e), and demyelination (Extended Data Fig. 10i,j).

To further assess T cell reactivity in humans, PBMCs of MS patients $(n=7)$ with elevated anti-EBNA1 and anti-GlialCAM titers were stimulated with EBNA1 and GlialCAM proteins and peptides and compared to PBMCs from healthy individuals. The CD4+ T cells trended towards more IFN- $y$ expression upon stimulation with EBNA1 ${ }_{A A 386-405}$ and GlialCAM ECD and ICD proteins, which resembled CD4+ T cells in mice (Extended Data Fig. 10k,I). In CD8+ T cells, high expression of IFN- $\gamma$ and granzyme-B indicated a robust $C D 8+T$ cell response against EBNA1 in both groups, while only CD8+ T cells from MS patients responded to GlialCAM ICD and GlialCAM ECD (Fig. 4f). One patient (MS16, Fig. 3o,p) showed extraordinarily high counts of IFN- -+ granzyme-B+ CD $8+T$ cells upon stimulation with EBNA1, GlialCAM ICD, and GlialCAM ${ }_{\text {AA370-389 }}$ (Extended Data Fig. 10m).

Together, we showed anti-GlialCAM antibodies are generated in response to EBNA1 ${ }_{\text {AA386- }}$ 405 immunization. Further, co-immunization of mice with EBNA1 ${ }_{\mathrm{AA} 386-405}$ and PLP $\mathrm{AA} 139-151$ enhanced CNS immune cell infiltration and demyelination, two prominent features of human MS pathology. In addition to anti-GlialCAM antibody titers, our human T cell data suggest an important role for CD8+ T cells in the immune response against GlialCAM.

\section{Discussion}

Viral triggers of MS and other autoimmune diseases have long been the subject of intense investigation, but evidence for their functional relevance is scarce ${ }^{8}$. By utilizing paired-chain BCR repertoire sequencing, rational selection of clonal antibody sequences, and three independent high-throughput proteomic 
platforms, we identified a monoclonal antibody from CSF of an MS patient that binds the MS-associated epitope EBNA1 ${ }_{A A 386-405}$ with high affinity, and cross-reacts with GlialCAM. We demonstrate the presence of cross-reactive EBV EBNA1 and GlialCAM antibodies in approximately $20-25 \%$ of MS patients, and show that co-immunization of mice with this EBNA1 epitope exacerbates autoimmune demyelination. Our findings demonstrate a mechanistic link between EBV infection and the pathobiology of MS.

Our analysis of the single-cell paired-chain BCR repertoire from blood and CSF of MS patients revealed features of intrathecal oligoclonal expansion with ongoing SHM, strikingly different from CSF BCR repertoires in other neuroinflammatory diseases. PB in the CSF of MS patients are highly clonal with longer CDR3 regions and skew towards use of five IGHV genes ${ }^{16-19,38,39}$. IGHV3-7 has been previously observed in MS CSF and OCB 17,19,39-41. The discovery that the IGHV3-7-based mAb MS39p2w174 crossbinds EBV EBNA1 and GlialCAM provides a direct link between IGHV3-7 and MS pathobiology.

Structural and binding data of MS39p2w174 from the CSF of an MS patient, and its unmutated germline ancestor show that germline only binds EBNA1 ${ }_{A A 486-405}$ with high affinity, and that SHM is required for high affinity cross-reactivity with GlialCAM. Due to the CNS-restricted expression of GlialCAM, it is likely that immature IGHV3-7 expressing B cells encoding a germline precursor of MS39p2w174 entered the CNS/CSF space, encountered GlialCAM antigen, and then underwent affinity maturation that generated clones encoding high affinity anti-GlialCAM antibodies. This is concordant with our BCR repertoire analysis, which revealed a highly clonal and skewed repertoire in CSF with low polyreactivity, indicative of a select group of B cells undergoing SHM, likely driven by perpetual activation by CNS GlialCAM.

The crystallographic structure revealed that Arg397 in EBNA1 ${ }_{\text {AA386-405 }}$ engages in two hydrogen-bonds with HC-CDR2 and is likely responsible for a significant part of the high binding affinity between MS39p2w174 and EBNA1. We show that phosphorylation of GlialCAM at Ser376 restores high affinity. Epitopes altered by PTMs have been described as targets for autoimmune responses, including citrullinated proteins in rheumatoid arthritis and phosphorylated Ro/La in systemic lupus erythematosus ${ }^{37,42}$. Tissue-specific differences of PTMs could contribute to a lack of central tolerance. Several kinases have been described as risk genes for MS, including MERTK, MAPK1 and 3, TYK2, potentially contributing to alternative phosphorylation patterns in the $\mathrm{CNS}^{43}$.

In addition, we show that co-immunization of mice with $E B N A 1_{A A 386-405}$ generates a robust $B$ cell response against GlialCAM and aggravates EAE. Our finding of activated PB in the CSF expressing exceptionally high levels of HLA-DR, suggest that these $B$ cells present antigens and exchange inflammatory signals with Tfh.

In summary, we present a detailed picture of the B cell repertoire in MS CSF and blood, demonstrating activated intrathecal IgG+ PBs undergoing affinity maturation. Analysis of a CSF-derived mAb targeting EBNA1 ${ }_{\text {AA386-405 }}$ revealed molecular mimicry to GlialCAM. These findings provide a mechanistic link between EBV infection and the pathobiology of MS. 


\section{References}

1. Cencioni, M. T., Mattoscio, M., Magliozzi, R., Bar-Or, A. \& Muraro, P. A. B cells in multiple sclerosis from targeted depletion to immune reconstitution therapies. Nat. Rev. Neurol. 17, 399-414 (2021).

2. Hauser, S. L. et al. Ocrelizumab versus Interferon Beta-1a in Relapsing Multiple Sclerosis. N. Engl. J. Med. 376, 221-234 (2017).

3. Bar-Or, A. et al. Epstein-Barr Virus in Multiple Sclerosis: Theory and Emerging Immunotherapies. Trends Mol. Med. 26, 296-310 (2020).

4. Jarius, S. et al. The MRZ reaction as a highly specific marker of multiple sclerosis: re-evaluation and structured review of the literature. J. Neurol. 264, 453-466 (2017).

5. Wang, Z. et al. Antibodies from Multiple Sclerosis Brain Identified Epstein-Barr Virus Nuclear Antigen 1 \& 2 Epitopes which Are Recognized by Oligoclonal Bands. J. Neuroimmune Pharmacol. 16, 567-580 (2021).

6. Thacker, E. L., Mirzaei, F. \& Ascherio, A. Infectious mononucleosis and risk for multiple sclerosis: a meta-analysis. Ann. Neurol. 59, 499-503 (2006).

7. Nielsen, T. R. et al. Effects of infectious mononucleosis and HLA-DRB $1 * 15$ in multiple sclerosis. Multiple Sclerosis Journal vol. 15 431-436 (2009).

8. Tengvall, K. et al. Molecular mimicry between Anoctamin 2 and Epstein-Barr virus nuclear antigen 1 associates with multiple sclerosis risk. Proc. Natl. Acad. Sci. U. S. A. 116, 16955-16960 (2019).

9. Ruprecht, K. et al. Multiple sclerosis: The elevated antibody response to Epstein-Barr virus primarily targets, but is not confined to, the glycine-alanine repeat of Epstein-Barr nuclear antigen-1. Journal of Neuroimmunology vol. 272 56-61 (2014).

10. Jafari, N. et al. No evidence for intrathecal lgG synthesis to Epstein Barr virus nuclear antigen-1 in multiple sclerosis. J. Clin. Virol. 49, 26-31 (2010).

11. Salzer, J. et al. Epstein-Barr virus antibodies and vitamin D in prospective multiple sclerosis biobank samples. Mult. Scler. 19, 1587-1591 (2013).

12. Sundqvist, E. et al. Epstein-Barr virus and multiple sclerosis: interaction with HLA. Genes Immun. 13, 14-20 (2012).

13. Ramesh, A. et al. A pathogenic and clonally expanded B cell transcriptome in active multiple sclerosis. Proc. Natl. Acad. Sci. U. S. A. 117, 22932-22943 (2020).

14. Kowarik, M. C. et al. Immune cell subtyping in the cerebrospinal fluid of patients with neurological diseases. J. Neurol. 261, 130-143 (2014). 
15. Tan, Y.-C. et al. Barcode-enabled sequencing of plasmablast antibody repertoires in rheumatoid arthritis. Arthritis rheumatol. 66, 2706-2715 (2014).

16. Palanichamy, A. et al. Immunoglobulin class-switched B cells form an active immune axis between CNS and periphery in multiple sclerosis. Sci. Transl. Med. 6, 248ra106 (2014).

17. von Büdingen, H.-C. et al. B cell exchange across the blood-brain barrier in multiple sclerosis. J. Clin. Invest. 122, 4533-4543 (2012).

18. Rounds, W. H. et al. MSPrecise: A molecular diagnostic test for multiple sclerosis using next generation sequencing. Gene 572, 191-197 (2015).

19. Bankoti, J. et al. In multiple sclerosis, oligoclonal bands connect to peripheral B-cell responses. Ann. Neurol. 75, 266-276 (2014).

20. Mescheriakova, J. Y., van Nierop, G. P., van der Eijk, A. A., Kreft, K. L. \& Hintzen, R. Q. EBNA-1 titer gradient in families with multiple sclerosis indicates a genetic contribution. Neurol Neuroimmunol Neuroinflamm 7, (2020).

21. Berman, H. M. et al. The Protein Data Bank. Acta Crystallogr. D Biol. Crystallogr. 58, 899-907 (2002).

22. Jeong, J. S. et al. Rapid Identification of Monospecific Monoclonal Antibodies Using a Human Proteome Microarray *. Mol. Cell. Proteomics 11, (2012).

23. Uhlén, M. et al. Proteomics. Tissue-based map of the human proteome. Science 347, 1260419 (2015).

24. Gilbert, A., Vidal, X. E., Estevez, R., Cohen-Salmon, M. \& Boulay, A.-C. Postnatal development of the astrocyte perivascular MLC1/GlialCAM complex defines a temporal window for the gliovascular unit maturation. Brain Struct. Funct. 224, 1267-1278 (2019).

25. Favre-Kontula, L. et al. GlialCAM, an immunoglobulin-like cell adhesion molecule is expressed in glial cells of the central nervous system. Glia 56, 633-645 (2008).

26. López-Hernández, T. et al. Mutant GlialCAM causes megalencephalic leukoencephalopathy with subcortical cysts, benign familial macrocephaly, and macrocephaly with retardation and autism. Am. J. Hum. Genet. 88, 422-432 (2011).

27. Han, M. H. et al. Proteomic analysis of active multiple sclerosis lesions reveals therapeutic targets. Nature 451, 1076-1081 (2008).

28. O'Donovan, B. et al. High-resolution epitope mapping of anti-Hu and anti-Yo autoimmunity by programmable phage display. Brain Commun 2, fcaa059 (2020). 
29. Bochkarev, A., Bochkareva, E., Frappier, L. \& Edwards, A. M. The 2.2 A structure of a permanganatesensitive DNA site bound by the Epstein-Barr virus origin binding protein, EBNA1. J. Mol. Biol. 284, 12731278 (1998).

30. Casciola-Rosen, L. A., Anhalt, G. \& Rosen, A. Autoantigens targeted in systemic lupus erythematosus are clustered in two populations of surface structures on apoptotic keratinocytes. Journal of Experimental Medicine vol. 179 1317-1330 (1994).

31. Pisetsky, D. S. \& Lipsky, P. E. New insights into the role of antinuclear antibodies in systemic lupus erythematosus. Nat. Rev. Rheumatol. 16, 565-579 (2020).

32. Schellekens, G. A. et al. The diagnostic properties of rheumatoid arthritis antibodies recognizing a cyclic citrullinated peptide. Arthritis Rheum. 43, 155-163 (2000).

33. Moh, M. C., Zhang, C., Luo, C., Lee, L. H. \& Shen, S. Structural and functional analyses of a novel iglike cell adhesion molecule, hepaCAM, in the human breast carcinoma MCF7 cells. J. Biol. Chem. 280, 27366-27374 (2005).

34. Britton, D. et al. Quantification of pancreatic cancer proteome and phosphorylome: indicates molecular events likely contributing to cancer and activity of drug targets. PLoS One 9, e90948 (2014).

35. Herskowitz, J. H. et al. Phosphoproteomic analysis reveals site-specific changes in GFAP and NDRG2 phosphorylation in frontotemporal lobar degeneration. J. Proteome Res. 9, 6368-6379 (2010).

36. Hornbeck, P. V., Chabra, I., Kornhauser, J. M., Skrzypek, E. \& Zhang, B. PhosphoSite: A bioinformatics resource dedicated to physiological protein phosphorylation. Proteomics 4, 1551-1561 (2004).

37. Zavala-Cerna, M. G. et al. The clinical significance of posttranslational modification of autoantigens. Clin. Rev. Allergy Immunol. 47, 73-90 (2014).

38. Bashford-Rogers, R. J. M., Smith, K. G. C. \& Thomas, D. C. Antibody repertoire analysis in polygenic autoimmune diseases. Immunology 155, 3-17 (2018).

39. Obermeier, B. et al. Matching of oligoclonal immunoglobulin transcriptomes and proteomes of cerebrospinal fluid in multiple sclerosis. Nat. Med. 14, 688-693 (2008).

40. Singh, V. et al. Cerebrospinal-fluid-derived Immunoglobulin G of Different Multiple Sclerosis Patients Shares Mutated Sequences in Complementarity Determining Regions *. Mol. Cell. Proteomics 12, 39243934 (2013).

41. Colombo, M. et al. Accumulation of clonally related B lymphocytes in the cerebrospinal fluid of multiple sclerosis patients. J. Immunol. 164, 2782-2789 (2000). 
42. Terzoglou, A. G., Routsias, J. G., Avrameas, S., Moutsopoulos, H. M. \& Tzioufas, A. G. Preferential recognition of the phosphorylated major linear B-cell epitope of La/SSB 349-368 aa by anti-La/SSB autoantibodies from patients with systemic autoimmune diseases. Clin. Exp. Immunol. 144, 432-439 (2006).

43. International Multiple Sclerosis Genetics Consortium et al. Genetic risk and a primary role for cellmediated immune mechanisms in multiple sclerosis. Nature 476, 214-219 (2011).

\section{Methods}

Study design and human Subjects. Patient samples were collected at Stanford University CA, USA, and the University of Heidelberg, Germany. Relapsing remitting MS (RRMS) was diagnosed according to the current McDonald criteria ${ }^{44,45}$. None of the patients met the diagnostic criteria for NMOSD, in particular spinal lesions spanning $\geq 3$ segments ${ }^{46}$. Patients were tested for antibodies against aquaporin- 4 and MOG and showed negative results. All included patients had elevated CSF white blood cell counts $\geq 10$ cells/ $\mu \mathrm{l}$, and blood contaminated CSF samples were excluded by visual and microscopic inspection. Paired peripheral blood and CSF samples were obtained th the time of clinical onset (clinically isolated syndrome, CIS) or during an acute relapse. All but one patient had not received any MS-specific treatment prior to sample collection (Extended Data Table 1). All experimental protocols were approved by the institutional review board of Stanford University (IRB\# 34529) and the ethics committee of the medical faculty of the University of Heidelberg (IRB\# S-466/2015). Written informed consent was obtained from each patient.

Cell preparation, antibody staining, and flow cytometric cell sorting. CSF was centrifuged immediately after lumbar puncture and cells were counted. PBMCs were isolated from heparin blood by density gradient centrifugation using Ficoll PLUS media (Cytiva). Cells were magnetically separated with antiCD19 magnetic beads (Dynabeads CD19 Pan B cell isolation kit, Invitrogen), then stained according to standard protocols, using antibodies against the following cell surface markers: CD20 (clone L27, dilution 1:10), CD38 (clone HB7, dilution 1:30), IgD (clone IA6-2, dilution 1:20) (all BD Biosciences), CD3 (clone OKT3, dilution 1:60), CD19 (clone HIB19, dilution 1:20), CD27 (clone 0323, dilution 1:20), IgM (clone MHM-88, dilution 1:40), HLA-DR (clone L243, dilution 1:100), a4 integrin (clone 9F10, dilution 1:100) (all BioLegend), IgA (clone IS11-8E10, dilution 1:20) (Miltenyi Biotec), and Sytox blue (ThermoFisher Scientific, dilution 1:500). Single cells were sorted with a FACSAria II cell sorter (BD Biosciences) using FACSDiva (v8.0, BD Biosciences) into 96-well PCR plates (BioRad). For single-cell repertoire sequencing, plasmablasts were sorted from PBMC (plasmablast gate (4.12\%) in panel 5 in the representative flow cytometry plots shown in Extended Data Fig. 1a). All B cells were sorted from CSF (B cell gate $(73.9 \%)$ in panel 4 in the representative flow cytometry plots shown in Extended Data Fig. 1b). FlowJo Version 10.7.1 (BD) and R version 3.6.1 was used to evaluate flow cytometry data.

Single-cell BCR repertoire sequencing. BCR repertoire sequencing was carried out using our in house developed plate-bound single-cell sequencing technology as described previously ${ }^{15,47,48}$. Briefly, reverse 
transcription with oligo-dT was carried out in separate wells, attaching unique well-ID barcodes by template switching activity of Maxima Reverse Transcriptase (ThermoFisher Scientific). Barcoded cDNA from each plate were pooled and amplified in 3 consecutive PCRs, including attaching plate-specific barcodes and sequencing adapters. PCRs were carried out separately for $\mathrm{HC}$ of $\lg G$, $\lg \mathrm{A}$, and $\lg \mathrm{M}$, as well as for $\mathrm{K}$ for $\lambda \mathrm{LC}$, and separate libraries were generated from each, gel-purified, cleaned with Ampure XP beads (Beckman Coulter), and sequenced on an Illumina MiSeq (Illumina) with $2 \times 330$ paired-end reads.

Sequence analysis. The MiSeq FASTQ workflow was used for Fastq generation and plate demultiplexing. $R$ version 3.6.1 was used for custom analyses. Paired reads of sequences that passed quality thresholds were stitched and separated by plate and well IDs. Similar reads sharing the same plate and well IDs were clustered into operational taxonomic units (OTUs) ${ }^{49}$. Consensus sequences were aligned to germline variable-chain immunoglobulin sequences with IMGT HighV-QUEST v1.3.1 ${ }^{50}$, which reports V, D, and J germline genes, $\mathrm{HC}$ and LC CDR3-lengths, and non-silent mutation counts and locations. Clonal expansions were defined based on sharing the same $\mathrm{HC}$ and LC $\mathrm{V}$ and $\mathrm{J}$ genes and exhibiting $>70 \%$ amino acid identity within the $\mathrm{HC}$ and LC CDR3s. Percent clonality represents the percent of all sequences that fulfill these criteria. To calculate IGHV, IGLV, IGHJ, and IGLJ gene usages, percent abundance of each particular gene was calculated in blood and CSF PB of each patient and means were calculated across patients. Genes that were present in less than three CSF samples were excluded from this analysis. While our sequencing method preferentially captures PB sequences due to higher amounts of immunoglobulin mRNA (Extended Data Fig. 2m), enough non-PB B cell sequences passed filter thresholds to compare the non-PB repertoire to the PB repertoire in 7 patients (Extended Data Fig. 2a,b). For patient samples MS12 and C6 only PB were captured (while gating on all B cells), and for MS39 only PB were sorted. For phylogenetic analysis, sequences were binned according to their $\mathrm{HC}$ V-gene family and V-gene. Concatenated LC and $\mathrm{HC}$ were then aligned with Muscle ${ }^{51}$ and clustered with PhyML ${ }^{52}$ using maximumlikelihood clustering. Each tree-partition was rooted by their HC V-gene. Phylogenetic trees were drawn in Python using the ETE 3 toolkit ${ }^{53}$.

Peptide identification with mass spectrometry. Immunoglobulins were purified from $1.5 \mathrm{ml}$ of CSF samples with Protein A (ThermoFisher Scientific). The purified IgGs were reduced with $0.02 \mathrm{M}$ dithiothreitol at $57^{\circ} \mathrm{C}$ for 1 hour, alkylated with $0.05 \mathrm{M}$ iodoacetamide at room temperature (RT) in the dark, and digested with trypsin overnight at RT. Peptides were extracted and desalted as previously described ${ }^{54}$. An aliquot of the peptide mixtures was loaded onto an Acclaim PepMap 100 precolumn $(75 \mu \mathrm{m} \times 2 \mathrm{~cm}, \mathrm{C} 18,3 \mu \mathrm{m}, 100 \AA)$ in-line with an EASY-Spray, PepMap column $(75 \mu \mathrm{m} \times 50 \mathrm{~cm}, \mathrm{C} 18,2 \mu \mathrm{m}$, $100 \AA ̊)$ with a $5 \mu \mathrm{m}$ emitter using the autosampler of an EASY-nLC 1000 (ThermoFisher Scientific). The peptides were gradient eluted into a Lumos Fusion Tribrid (ThermoFisher Scientific) mass spectrometer using a $120 \mathrm{~min}$ gradient from $5 \%$ to $35 \%$ solvent B (90\% acetonitrile, $0.5 \%$ acetic acid), followed by 10 minutes from $35 \%$ to $45 \%$ solvent $B$ and $10 \mathrm{~min}$ from 45 to $100 \% \mathrm{~B}$. High resolution full MS spectra were acquired with a resolution of 120,000 , an AGC target of $4 \times 10^{5}$, a maximum ion time of $50 \mathrm{~ms}$, and scan range of 400 to $1800 \mathrm{~m} / \mathrm{z}$. Following each full MS scan as many data-dependent HCD MS/MS spectra were acquired in the orbitrap as possible in a 3 second cycle time. Monoisotopic precursor selection

Page 15/31 
(MIPS) was set to peptide, precursors with a charge state of $2-7$ and minimum intensity of $5 \times 10^{4}$ were selected for MS/MS. Dynamic exclusion was set to 60 seconds after a single selection. All MS/MS spectra were collected using the following instrument parameters: resolution of 30,000, an AGC target of $10^{5}$, maximum ion time of $120 \mathrm{~ms}$, two microscans, $1.6 \mathrm{~m} / \mathrm{z}$ isolation window, and Normalized Collision Energy (NCE) of 32.

The MS/MS spectra were searched against the respective peptide specific database including common contaminant proteins using the search engine Byonic ${ }^{54}$. The search parameters were set to trypsin allowing two missed cleavages, fixed modification of carbamidomethyl on cysteine, variable modification of oxidation on methionine and deamidation on glutamine and asparagine. Peptides mapping to variable regions of IgG were manually verified. In order to include only sequence-specific peptides, peptides that aligned to non-immunoglobulin or constant-region sequences were excluded from the analysis, as were peptides that aligned to the repertoire of multiple patients. Included were peptides that aligned to one variable sequence in a single patient. Peptides that aligned to more than one variable sequence in a single patient were included if all matching sequences were exact matches or clonally related, in which case the peptide was counted as representative for all matches. Counts of identical or non-identical peptide spectral matches (PSM) per sequence were tallied for each sequence. Sequences that had $>1$ or $>10$ matching peptides were presented as percentage of all sequences (Fig. 1j,k, Extended Data Fig. 2g,h). The mass spectrometry files are accessible at MassIVE (massive.ucsd.edu) under accession number MSV000086829.

Selection and recombinant expression of mAbs. Representative antibodies from the largest clonal B cell expansions in the CSF of each patient were selected for recombinant expression. In patients with more than 10 large clonal expansions, sequences were preferentially chosen based on their usage of one of the 11 most abundant IGHV genes in the CSF (Extended Data Fig. 3). HC and LC variable sequences were custom generated (IDT), and cloned into pFuse vectors (Invivogen), containing human IgG constant region or kappa or lambda constant regions, respectively. Fab HC were expressed in in-house plasmids, containing the constant-region $\mathrm{C} 1$ up to Cys103. Plasmids were transfected into Expi293T cells using Expifectamine (ThermoFisher Scientific). Culture medium was harvested after 4 and 7 days post transfection. mAbs and Fabs were purified with protein $A$ and protein $G$ resins, respectively (ThermoFisher Scientific). Antibody concentrations were measured with a nanodrop spectrophotometer (ThermoFisher Scientific) and hlgG quantitation ELISAs (Bethyl Laboratories) and checked for purity on SDS protein gels with Coomassie staining.

Protein expression and purification. EBNA1 proteins and peptides were obtained from: full-length AA1-641 (Abcam), AA328-641 (Virion Serion), AA408-641 (ProspecBio). GlialCAM proteins and peptides: full-length AA34-416 (OriGene), ECD AA34-234 (Novoprotein), and ICD AA262-416 with N-terminal His-Tag was cloned into a pet30(+) vector, expressed in BL21 chemically competent E. coli (Sigma Aldrich) to an OD of $600 \mathrm{~nm}$, and induced with IPTG (Sigma Aldrich) for $3 \mathrm{~h}$ at $37^{\circ} \mathrm{C}$. Cell pellets were disrupted by sonication and proteins were purified with cOmplete His-Tag Purification Resins (Roche Life Science), followed by 
size-exclusion purification (Cytiva). For all other used peptides and proteins see Supplementary Tables 24.

Planar protein microarrays. Protein microarrays were generated as described previously ${ }^{55,56}$ (https://web.stanford.edu/group/antigenarrays/). In brief, peptides, recombinant proteins, and lysates were diluted at the indicated concentrations in a 1:1 solution of PBS/water and protein printing buffer (Arraylt) (Supplementary Tables 2-4), aliquoted on 384-well plates, and printed on SuperEpoxy Slides using a NanoPrint LM210 system (Arraylt). Two independent quadruplicates of each analyte were spotted, and some proteins were used in several versions / preparations from different sources (Supplementary Table 2). Ready-made HuProt Arrays version 3.1 were obtained from CDI labs. Arrays were circumscribed with a hydrophobic marker, blocked overnight at $4^{\circ} \mathrm{C}$ in PBS containing $3 \%$ FCS and $0.1 \%$ Tween-20, and incubated with individual mAbs at a concentration of $1 \mu \mathrm{g} / \mathrm{ml}$ for $1 \mathrm{~h}$ at $4^{\circ} \mathrm{C}$, then washed twice for $20 \mathrm{~min}$ in blocking buffer on a rotating shaker. Arrays were then incubated with Cy-3-conjugated secondary goat anti-human IgG $(0.8 \mu \mathrm{g} / \mathrm{mL})$ (Jackson ImmunoResearch) for $1 \mathrm{~h}$ at $4{ }^{\circ} \mathrm{C}$, then washed twice for $30 \mathrm{~min}$ in blocking buffer, twice for $30 \mathrm{~min}$ in PBS, and twice for $15 \mathrm{~s}$ in water. Arrays were spun dry and scanned with a GenePix 4000B scanner (Molecular Devices). Median pixel intensities for each fluorescent spot were determined with GenePix Pro-3.0 software (Molecular Devices). Z-scores for each row of antigens were calculated for viral antigens, raw intensities were analyzed for GlialCAM arrays. Heatmaps were generated with Morpheus software (The Broad Institute; https://software.broadinstitute.org/morpheus).

PhIP-Seq. PhIP-Seq was performed using a human proteome-wide library expressing overlapping 49amino-acid peptides with a 24-AA sliding window approach starting at the N-terminus. Briefly, 2uL $(1 \mathrm{ug} / \mathrm{mL})$ of substrate antibody was diluted 1:100 in blocking buffer for two sequential rounds of immunoprecipitation. After the second round of immunoprecipitation and amplification in E. coli, next generation sequencing libraries were prepared for paired-end 150 base next generation DNA sequencing on the Illumina Hi-Seq platform as previously described ${ }^{28,57}$. After alignment of the reads to the reference peptide sequences, quality control was performed and only reads present at an abundance of at least 10 reads per hundred thousand were carried forward. The number of reads mapping to each peptide were then counted and individually scored as a percentage of the total.

ELISA. Cytokine ELISA kits were used according to manufacturers' instructions: mouse IL-6, IL-10, IL-12, IFN- $\gamma$, and TNF (BD Biosciences), and IL-17A (ThermoFisher Scientific). For protein and peptide ELISAs, MaxiSorp 384-well plates (ThermoFisher Scientific) were coated with $1 \mu \mathrm{g} / \mathrm{ml}$ peptide or protein in carbonate-bicarbonate buffer at $4^{\circ} \mathrm{C}$ overnight, then washed $6 x$ with PBST (PBS $+0.05 \%$ Tween 20 ), blocked with blocking buffer (PBS $+1 \%$ BSA) for $1 \mathrm{~h}$, and mAbs were applied at $1 \mu \mathrm{g} / \mathrm{ml}$ in blocking buffer. Human and mouse plasma samples were diluted 1:100 and $T$ cell supernatants 1:4 in blocking buffer. After overnight incubation at $4^{\circ} \mathrm{C}$, plates were washed again $6 \mathrm{x}$ with PBST, secondary antibody HRP-conjugated goat anti-human IgG (Jackson ImmunoResearch), was applied for $1 \mathrm{~h}$ at RT, and after 6 additional washes with PBST, plates were developed with TMB substrate (ThermoFisher Scientific), stopped with $1 \mathrm{~N}$ sulfuric acid, and read on a SpectraMax Paradigm Microplate Reader (Molecular 
Devices). For plasma ELISAs with blocking of plasma IgG, MaxiSorp 384-well plates were coated with $2 \mu \mathrm{g} / \mathrm{ml}$ recombinant Protein $\mathrm{G}$ (Acro Biosystems) at $4^{\circ} \mathrm{C}$ over night, then washed $6 \mathrm{x}$ with PBST, and incubated with 1:100 diluted plasma at $4^{\circ} \mathrm{C}$ over night. Plates were again washed $6 x$ with PBST, and then incubated with the respective blocking peptides at $10 \mu \mathrm{g} / \mathrm{ml}$ for $2 \mathrm{~h}$ at RT. Then, biotinylated EBNA1 ${ }_{A A 385-}$ 405 was added at $1 \mu \mathrm{g} / \mathrm{ml}$ and incubated for $1 \mathrm{~h}$ at RT. Plates were washed again $6 \mathrm{x}$ with PBST, incubated with HRP-conjugated streptavidin (BioLegend) for $1 \mathrm{~h}$ at RT, and developed with TMB substrate as described above.

Western blotting. Western blots were run according to standard protocols. Briefly, purified proteins were boiled in Laemmli-buffer with $10 \%$ beta-mercaptoethanol for 5 minutes, run on $4-12 \%$ Criterion XT Bis-Tris protein Gels (Bio-Rad), then transferred onto a nitrocellulose membrane using a Trans-Blot Turbo semi-dry transfer system (Bio-Rad) and stained with MS39p2w174 at 10 $\mathrm{g} / \mathrm{ml}$ or with mouse anti-EBNA1 antibody (Biorbyt) or mouse anti-GlialCAM antibody (R\&D Systems) followed by secondary HRP-conjugated goat anti-human IgG and anti-mouse IgG (Jackson ImmunoResearch). Coomassie gels were run concomitantly, fixed with $10 \%$ methanol and $7 \%$ acetic acid, and stained according to standard protocols. Uncropped western blot and Coomassie images are available in Supplementary Figure 1.

\section{Fluorescent Immunohistochemistry on mouse brain slices and Immunofluorescence on primary cultured}

rat oligodendrocytes. An adult mouse (F1 generation of FVB x C57blk6 cross) was transcardially perfused with $4 \%$ paraformaldehyde and post-fixed in 4\% PFA overnight at 4C. After sucrose equilibration, the brain was blocked in OCT and sectioned at $12 \mu \mathrm{m}$ on a standard cryostat. Sections were permeabilized and blocked in PBS containing $10 \%$ lamb serum and $0.1 \%$ triton $x-100$. Sections were immunostained with concomitantly expressed control mAb anti-DSG3 (Acc\#: HQ338093.1 and HQ338094.1) $(18 \mu \mathrm{g} / \mathrm{mL}$ ), MS39p2w174 $(18 \mu \mathrm{g} / \mathrm{mL})$, or PBS in blocking buffer overnight at 4C. Sections were washed five times with PBS over 1 hour and counterstained with anti-human IgG for 1 hour at room temperature (Alexa Fluor 488, Jackson ImmunoResearch) $(2 \mu \mathrm{g} / \mathrm{mL})$. Nuclei were stained with DAPI at 1:2000 for 5 minutes at room temperature. Rat oligodendrocyte precursor cells were prepared from rat embryos followed by panning and in-vitro differentiation into primary rat oligodendrocytes ${ }^{58}$. Cells on cover slips were permeabilized with ice-cold $100 \%$ methanol for $10 \mathrm{~min}$, blocked with $10 \%$ donkey serum for $1 \mathrm{~h}$ at RT, and then stained with isotype control (anti-DSG3) or MS39p2w174 at $10 \mu \mathrm{g} / \mathrm{ml}$ in $1 \%$ donkey serum for $1 \mathrm{~h}$ at RT, before incubation with secondary Alexa Fluor 647 donkey anti-human IgG antibody (Jackson ImmunoResearch) for $1 \mathrm{~h}$ at RT. Confocal images were taken with a Zeiss LSM 880 confocal microscope using ZEN software (Zeiss).

Bio-Layer Interferometry. Association and dissociation constants of mAbs to proteins and peptides were measured with bio-layer interferometry on an Octet QK device (Fortebio / Sartorius, Fremont, CA) according to standard protocols. For peptide kinetics, biotinylated peptides were bound to high precision streptavidin (SAX) biosensors (peptide concentration in solution: $100 \mathrm{nM}$ ) and mAbs MS39p2w174 and germline were probed as analytes in concentrations ranging from $10-270 \mathrm{nM}$. For protein kinetics, mAbs were bound to anti-hlgG Fc Capture (AHC) biosensors (mAb concentration in solution: $20 \mathrm{nM}$ ), and proteins were probed as analytes in concentrations ranging from $1.56-125 \mathrm{nM}$. Data was analyzed with 
BLI analysis software (Fortebio / Sartorius, version 7.1) and with GraphPad prism (version 8.4). Buffer controls were subtracted, and curves were fitted globally for each group consisting of all concentrations of the same ligand. $K_{D}$ values $\pm S D$ as well as association / dissociation curves were reported and plotted with GraphPad prism (version 8.4). $\mathrm{K}_{\mathrm{D}}, \mathrm{K}_{\mathrm{on}}$, and $\mathrm{K}_{\text {off }}$ values are shown in Supplementary Table 6.

Prediction of Protein Disorder. Order and disorder along the amino acid sequences of EBNA1 and GlialCAM were analyzed with PONDR (Predictor of Natural Disordered Regions, WSU Research Foundation $)^{59}$, using the VSL2 algorithm.

Crystallization of antibody-antigen complexes. EBNA $1_{\text {AA386-405 }} 20$ mer peptides ( $>98 \%$ purity) (Sigma Aldrich) were mixed with MS39p2w174-Fab $(15 \mathrm{mg} / \mathrm{ml})$ in a 1:7.5 molar ratio and incubated overnight. Crystals for MS39p2w174-Fab + EBNA1 ${ }_{\text {AA386-405 }}$ grew in 0.48M Sodium Citrate, 0.72M Sodium/Potassium Phosphate, and 3\% MPD (v/v) in 0.1M HEPES, pH 6.9 (Extended Data Fig. 6c). Crystals were harvested, cryo-protected with a quick dip in a cryo-solution containing the well solution with $25 \%$ glycerol, and flash cooled in liquid nitrogen. Data were collected at beamline SSRL 12-2 at the Stanford Synchrotron Radiation Lightsource, SLAC National Accelerator Laboratory, and processed and scaled using XDS/aimless and Staraniso ${ }^{60,61}$. Crystals belonged to space group $1222(a=119.66 \AA, b=$ $137.56 \AA, c=179.00 \AA, a=\beta=\gamma=90^{\circ}$ ) and contained two Fab-peptide complexes per asymmetric unit (Extended Data Fig. 6d). Phaser was used for molecular replacement ${ }^{62}$ with the model structure 4LRI $(\mathrm{PDB})^{63}$, stripped of all CDR loops. Loops were re-constructed with Coot ${ }^{64}$ and structures were refined with phenix.refine ${ }^{65,66}$ in iterations with Coot. Measurements and figure design was done with Pymol v2. ${ }^{67}$. The structure was deposited in the protein data bank (PDB, www.rcsb.org ${ }^{26}$ ) with PDB ID: 7K7R.

Mouse Immunization, EAE, and histology. All animal experiments were performed in accordance with state and federal guidelines and regulations, and approved by the Stanford Institutional Animal Care and use Committee. 8-week-old female SJL/J and FVB x C57BL/6 mice were purchased from The Jackson Laboratory. The mice were housed in recyclable individually ventilated (IVC) cages, with a 12-light/hour dark cycle, at a temperature of 70 degrees $F$, and with $50 \%$ humidity. Mice were immunized s.c. with 200

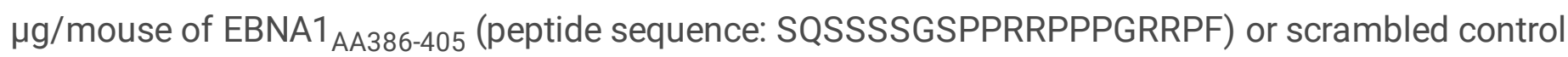
peptide (peptide sequence: SPSRPGRSRSRGSPFPQPSP) (10 mice per group), mixed with $100 \mu \mathrm{g} / \mathrm{mouse}$ of CpG (ODN1826, Invivogen, San Diego, CA) in $100 \mu \mathrm{l} /$ mouse incomplete Freund's adjuvant (BD Difco, Franklin Lakes, NJ). 3 weeks later EAE was induced by s.c. immunization with $200 \mu \mathrm{g} /$ mouse of

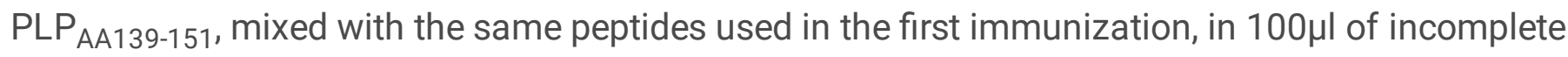
Freund's adjuvant, supplemented with $200 \mu \mathrm{g}$ /mouse of mycobacterium tuberculosis (strain H37 RA, BD Difco). Serum samples were obtained by retro-orbital blood draws 3 days before the 1 st and 2nd immunizations (day -24 and day -3 ), and during termination of the experiment (day 50). Mice were weighed daily, and disease severity was assessed according to a 5-point standard scoring system: 0 , no clinical signs; 1 , loss of tail tone; 2 , hind limb weakness; 3 , complete hind limb paralysis; 4 , hind limb and forelimb paralysis; 5 , moribund or dead. Mice were euthanized on day 50 post induction of EAE by deep anesthesia with i.p. injections of $0.01 \mathrm{ml} / \mathrm{g}$ body weight 7.2\% Xylazine (Bayer Healthcare, Leverkusen, 
Germany) and 10.8\% Ketamine (Pfizer, New York City, NY). Lymph nodes and spleens were extracted, and mice were then perfused with $10 \mathrm{ml}$ PBS and $20 \mathrm{ml}$ 4\% paraformaldehyde (PFA) (Electron Microscopy Sciences, Hatfield, PA). Brains and spinal cords were extracted, stored in 4\% PFA overnight, followed by $30 \%$ sucrose in PBS. Tissues were embedded in paraffin, sectioned, and stained for H\&E as well as Luxol fast blue according to standard protocols. Infiltration of inflammatory cells into the spinal cord on H\&E slides was assessed by a blinded investigator by counting lesions of infiltrating cells per slide, taking lesion size into account: 1: small infiltrate ( $<10$ cells), 2: medium infiltrate ( $<100$ cells), 3: large infiltrate ( $>100$ cells). Demyelination was assessed by a blinded investigator according to a histological score ${ }^{64}$ : 0.5 , single demyelinated spot; 1 , several spots; 2 , confluent sites of demyelination; 3 , extensive demyelination, less than half of a spinal cord; 4, demyelination of more than half of the spinal cord; and 5 , extensive demyelination affecting $>85 \%$ of the total white matter of the spinal cord.

In vitro human T cell stimulation with proteins and peptides. Cryopreserved PBMCs were thawed and stabilized overnight at $37^{\circ} \mathrm{C}$. The cells were pre-incubated for 30 minutes at $37^{\circ} \mathrm{C}, 5 \% \mathrm{CO}_{2}$ with Polymyxin $B$ (Sigma Aldrich) at a concentration of $10 \mathrm{mg} / \mathrm{mL}$. The cells were then incubated for 16 hours with $100 \mathrm{mM}$ of each recombinant protein or peptide in the presence of $2 \mathrm{mg} / \mathrm{ml}$ of anti-human CD28 (clone CD28.2, BD Biosciences) and anti-CD49d (clone 9F10, BioLegend) antibodies and IL-2 (50 IU/ml, Peprotech, Cranbury, NJ) and IL-7 (5 ng/ml, Peprotech). To detect intracellular staining, eBioscience ${ }^{\mathrm{TM}}$ Protein Transport Inhibitor Cocktail (500X, ThermoFisher Scientific) was added during the final 5 hours of culture. After 16 hours, the cells were labeled with Fixable Viability Stain 510 (BD Biosciences) for live cell staining and fluorophore conjugated anti-CD3 (clone SK7, BD Biosciences), CD4 (clone RPA-T4, BD Biosciences), CD8 (clone RPA-T8, BD Biosciences), Granzyme B (clone GB11, BD Biosciences), IFNg (clone B27, BD Biosciences) and IL-17A (clone BL168, BD Biosciences) antibodies and detected using a BD LSR Fortessa.

Data analysis and statistics. The publicly available dataset from Han et al. ${ }^{27}$ was searched for abundance of GlialCAM in MS lesions. The old accession number Q8N7I3 was found, which was annotated in 2008 as unknown hypothetical protein and has since been replaced by accession number Q14CZ8. GlialCAM was identified with 2.5 mean spectral counts (MSC) in control tissue, 1.3 MSC in chronic plaques, 1.8 MSC in acute plaques, and $8 \mathrm{MSC}$ in chronic-active plaques.

GraphPad Prism version 8.4.1 and R version 3.6.1 were used for statistical analyses. Statistical tests used are indicated in the respective methods section or in the figure legends.

\section{Supplementary References}

44. Thompson, A. J. et al. Diagnosis of multiple sclerosis: 2017 revisions of the McDonald criteria. Lancet Neurol. 17, 162-173 (2018).

45. Polman, C. H. et al. Diagnostic criteria for multiple sclerosis: 2010 revisions to the McDonald criteria. Ann. Neurol. 69, 292-302 (2011). 
46. Wingerchuk, D. M. et al. International consensus diagnostic criteria for neuromyelitis optica spectrum disorders. Neurology 85, 177-189 (2015).

47. Tan, Y.-C. et al. High-throughput sequencing of natively paired antibody chains provides evidence for original antigenic sin shaping the antibody response to influenza vaccination. Clin. Immunol. 151, 55-65 (2014).

48. Blum, L. K. et al. Circulating plasmablasts are elevated and produce pathogenic anti-endothelial cell autoantibodies in idiopathic pulmonary arterial hypertension. Eur. J. Immunol. 48, 874-884 (2018).

49. Edgar, R. C. UPARSE: highly accurate OTU sequences from microbial amplicon reads. Nat. Methods 10, 996-998 (2013).

50. Alamyar, E., Duroux, P., Lefranc, M.-P. \& Giudicelli, V. IMGT(®) tools for the nucleotide analysis of immunoglobulin (IG) and T cell receptor (TR) V-(D)-J repertoires, polymorphisms, and IG mutations: IMGT/V-QUEST and IMGT/HighV-QUEST for NGS. Methods Mol. Biol. 882, 569-604 (2012).

51. Edgar, R. C. MUSCLE: multiple sequence alignment with high accuracy and high throughput. Nucleic Acids Res. 32, 1792-1797 (2004).

52. Guindon, S. et al. New algorithms and methods to estimate maximum-likelihood phylogenies: assessing the performance of PhyML 3.0. Syst. Biol. 59, 307-321 (2010).

53. Huerta-Cepas, J., Serra, F. \& Bork, P. ETE 3: Reconstruction, Analysis, and Visualization of Phylogenomic Data. Mol. Biol. Evol. 33, 1635-1638 (2016).

54. Bern, M., Kil, Y. J. \& Becker, C. Byonic: advanced peptide and protein identification software. Curr. Protoc. Bioinformatics Chapter 13, Unit13.20 (2012).

55. Robinson, W. H. et al. Autoantigen microarrays for multiplex characterization of autoantibody responses. Nat. Med. 8, 295-301 (2002).

56. Kuerten, S. Autoantibodies against central nervous system antigens in a subset of B cell-dominant multiple sclerosis patients. Proc. Natl. Acad. Sci 202011249, 2011249117 (2020).

57. Schubert, R. D. et al. Pan-viral serology implicates enteroviruses in acute flaccid myelitis. Nat. Med. $25,1748-1752$ (2019).

58. Emery, B. \& Dugas, J. C. Purification of oligodendrocyte lineage cells from mouse cortices by immunopanning. Cold Spring Harb. Protoc. 2013, 854-868 (2013).

59. Obradovic, Z. et al. Predicting intrinsic disorder from amino acid sequence. Proteins 53 Suppl 6, 566572 (2003). 
60. Evans, P. R. \& Murshudov, G. N. How good are my data and what is the resolution? Acta Crystallogr. D Biol. Crystallogr. 69, 1204-1214 (2013).

61. Tickle, I. STARANISO: use of a WebGL-based 3D interactive graphical display to represent and visualise data quality metrics for anisotropic macromolecular diffraction data. Acta Crystallogr. A Found. Adv. 75, e162-e162 (2019).

62. McCoy, A. J. et al. Phaser crystallographic software. J. Appl. Crystallogr. 40, 658-674 (2007).

63. Fouts, A. E. et al. Mechanism for neutralizing activity by the anti-CMV $\mathrm{gH} / \mathrm{gL}$ monoclonal antibody MSL-109. Proc. Natl. Acad. Sci. U. S. A. 111, 8209-8214 (2014).

64. Lanz, T. V. et al. Tryptophan-2,3-Dioxygenase (TDO) deficiency is associated with subclinical neuroprotection in a mouse model of multiple sclerosis. Sci. Rep. 7, 41271 (2017).

\section{Declarations}

Acknowledgments: We thank Wolfgang Wick for support with sample collection, Ben Bell for advice on crystallization, George Harauz for insightful discussions on poly-proline motifs, and the staff at SSRL for assistance with data collection. Funding: This work was supported by NIH R01 AR063676 and U19 Al110491 to W.H.R., the Juvenile Diabetes Research Foundation and Lupus Research Alliance Funding to W.H.R. and T.V.L., the German Research Foundation (DFG, LA3657/1) to T.V.L., Atara to L.S. and P.P.H., and the German Research Foundation to M.P. (DFG, project 406052676; PL-315/5-1). The mass spectrometric experiments are in part supported by the NYU Grossman School of Medicine and a shared instrumentation grant NIH 1S100D010582-01A1. Use of the Stanford Synchrotron Radiation Lightsource, SLAC National Accelerator Laboratory, is supported by the U.S. Department of Energy, Office of Science, Office of Basic Energy Sciences under Contract No. DE-AC02-76SF00515. The SSRL Structural Molecular Biology Program is supported by the DOE Office of Biological and Environmental Research, and by $\mathrm{NIH}$ GIGMS P30GM133894. The contents of this publication are solely the responsibility of the authors and do not represent the official views of NIGMS or $\mathrm{NIH}$.

Author contributions: Conceptualization, T.V.L., L.S., W.H.R.; Methodology, T.V.L., R.C.B., P.P.H., K.M.J., D.F., R.A.F., A.M.G., R.D.S., B.T., V.C.C., B.M.U., J.-S.M., M.I., J.B.Z.; Software, T.V.L., R.C.B., D.F., K.M.J., V.C.C.; Validation, T.V.L., R.C.B., K.M.J., B.M.U., R.J.M.B.-R., R.A.S., K.C.G., L.S., W.H.R., Formal Analysis, T.V.L., R.C.B., K.M.J., D.F., A.M.G., C.M.B., V.C.C., B.M.U.; Investigation, T.V.L., R.C.B., P.P.H., D.F., G.-S.N., C.M.B., R.D.S., I.A.H., S.E.V., B.T., V.C.C., J.-S.M., M.I.; Resources, T.V.L., P.P.H., D.F., B.T., J.E.D., C.B.L., L.B.K., B.M.U., M.R.W., M.S.A., J.L.D., M.P., R.A.S., K.C.G., L.S., W.H.R.; Data Curation, T.V.L., R.C.B., K.M.J., D.F., V.C.C., B.M.U.; Writing - Original Draft, T.V.L.; Writing - Review \& Editing, T.V.L., R.C.B., P.P.H., L.S., W.H.R.; Visualization, T.V.L., R.C.B., K.M.J.; Supervision, T.V.L., W.H.R.; Project Administration, T.V.L., W.H.R.; Funding Acquisition, T.V.L., P.P.H., B.T.A., M.P., L.S., W.H.R. 
Competing interests: W.H.R. owns equity in, serves as a consultant to, and is a member of the Board of Directors of Atreca, Inc. L.S. owns equity in and serves as a consultant to Atreca, Inc. T.V.L. and W.H.R. are coinventors on a patent application filed by Stanford University that includes antibodies to EBV. The remaining authors declare no competing interests.

Data availability statement: The genomic datasets analyzed during the study have been uploaded to SRA, Accession \#: PRJNA780931. Mass spectrometry data is available at www.massive.ucsd.edu Accession \#: MSV000086829. Structural data is available at www.rcsb.org PDB ID: 7K7R. Requests for data and materials should be addressed to tlanz@stanford.edu or wrobins@stanford.edu.

\section{Figures}




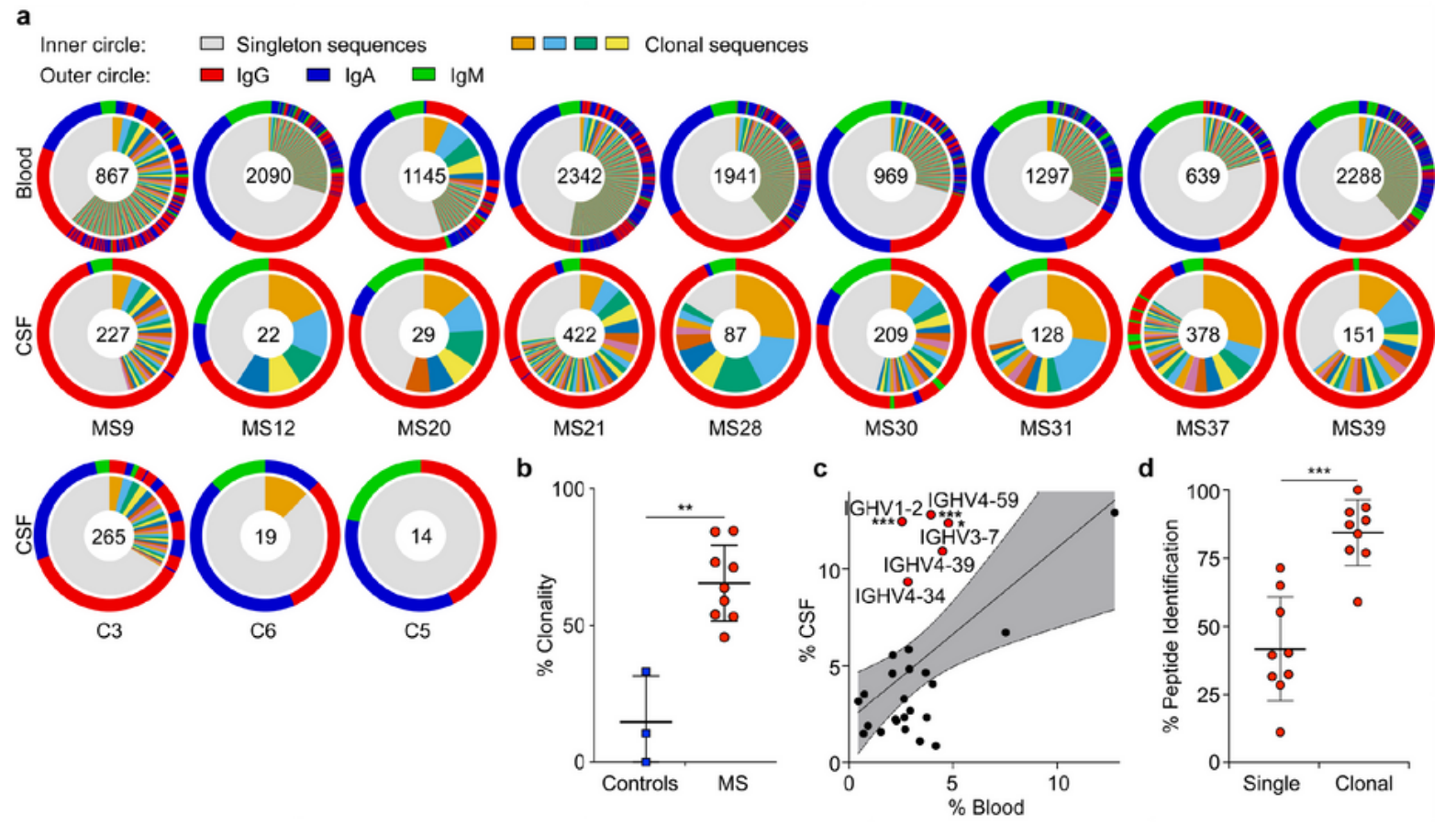

\section{Figure 1}

B cell repertoires in MS blood and CSF. a-c, Single-cell BCR repertoire sequencing data, a, individual repertoires from paired blood plasmablasts (top row) and CSF B cells (second row) of MS patients and CSF B cells of control patients (bottom row) ( $n=9 \mathrm{MS}$ patients, $n=3$ control patients), numbers indicate number of sequences, inner circle: colored wedges represent clonal expansions and grey area represents singleton antibody sequences, outer circle: immunoglobulin classes, red: IgG, blue: IgA, green: $\lg M$, 
sequence locations in outer circle correspond to inner circle. b, Clonality analysis, percent of clonal sequences in CSF B cells are shown, comparing BCR repertoires of control patients $(n=3)$ to MS patients $(n=9)$. Means \pm SD of each group are shown. ${ }^{*} P=0.0091$, two-tailed Mann-Whitney test. C, IGHV gene distribution in blood vs. CSF plasmablasts, each dot represents the usage of one IGHV gene across $n=9$ MS patient repertoires in the respective compartments. Linear regression line and $95 \%$ confidence interval is shown, IGHV1-2, $* \star \star P=5.6 \times 10^{-4}$; IGHV4-59, $* \star * P=9.2 \times 10^{-4} ;$ IGHV3-7, $* P=0.025$, unpaired two-tailed Student's t tests, Holm-Sidak corrected for multiple comparisons. d, Mass spectrometry data of purified CSF immunoglobulins, showing variable chain sequences that were uniquely identified with mass spectrometry in the singleton BCR sequences vs. clonal sequences (peptide-spectral matches (PSM) cutoff $\geq 1$ ), means \pm SD of $n=9$ MS patients. ${ }^{\star \star \star} P=0.0002$, two-tailed Mann-Whitney test. 

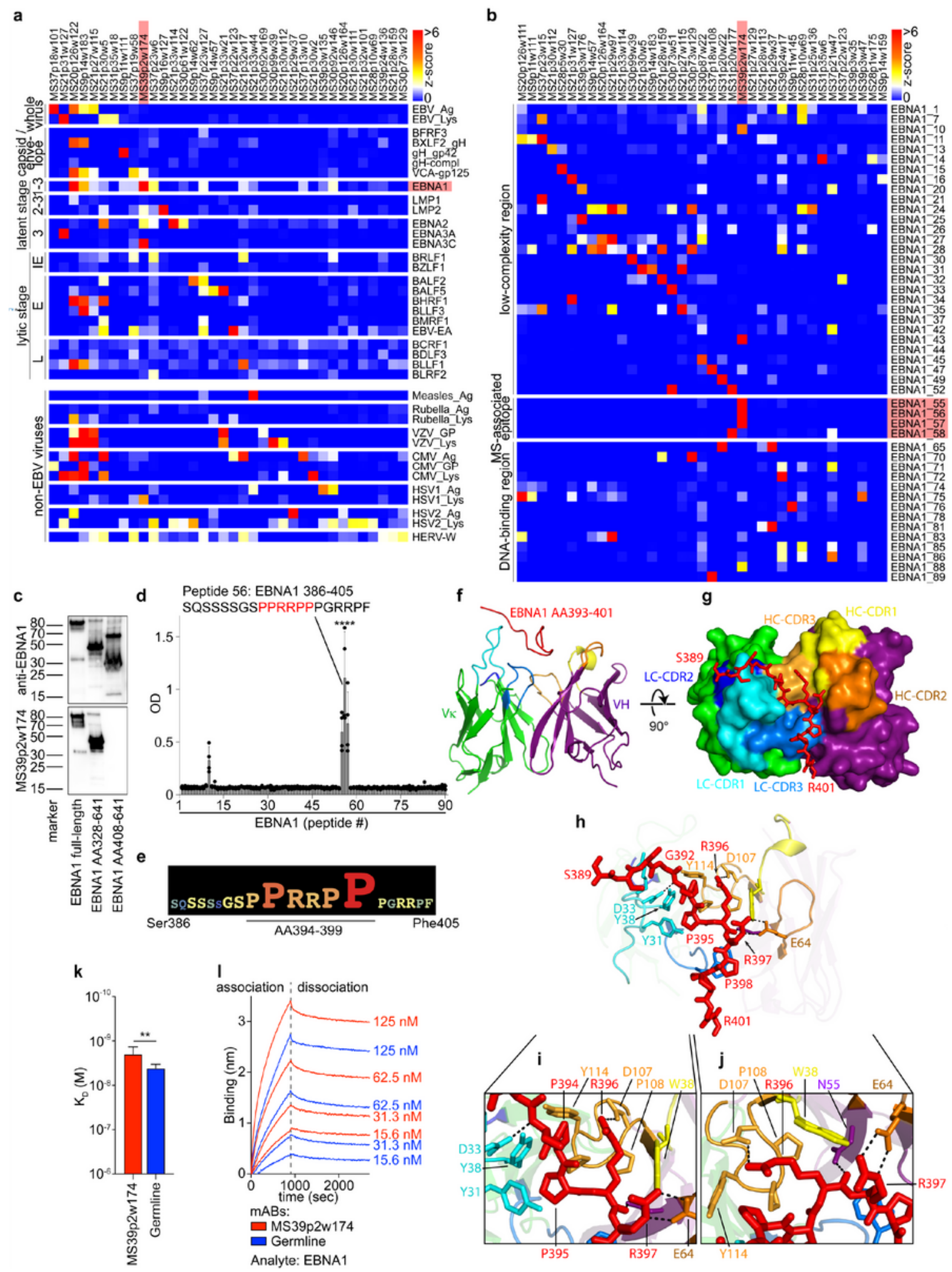

Figure 2

MS CSF B cell mAb reactivity to EBV proteins and interaction of MS39p2w174 with EBNA1 ${ }_{A A 386-405} \cdot a$, Protein microarray data showing MS CSF mAb reactivities (z-scores) to viral lysates and EBV proteins, $\mathbf{b}$, MS CSF mAB reactivities to EBNA1 peptides. Selected mAbs with highest reactivities are shown ( $n=36$ (a) and $n=37$ (b) out of $n=148$ ). Data from one experiment carried out in 8 technical replicates. IE: immediate early, E: early, and L: late lytic stage, red: mAb MS39p2w174 and antigen EBNA1 / MS-associated region. 
c, Western blot analysis of recombinant EBNA1 (full-length and truncated proteins), stained on separate blots with commercial anti-C-terminal EBNAI antibody (top panel) and MS39p2w174 (bottom panel), molecular weight marker in kDa. d,e, ELISA data, d, overlapping peptide scan of MS39p2w174 binding EBNA1 peptides (20mers, 13AA overlap), means \pm SD of $n=4$ independent experiments, each measured in duplicates. e, Alanine-scan, EBNA1 ${ }_{A A 386-405}$ logo representation showing the contribution of each residue to binding of MS39p2w174. $\mathbf{f - j}$, Crystal structure of MS39p2w174 in complex with EBNA1 AA386-405. f, Cartoon representation, showing EBNA1 ${ }_{A A 393-401}$ in the binding groove. Peptide (red), HC (green/blue), LC (purple/yellow/orange), CDR loop colors correspond to annotations in $(\mathbf{g}) . \mathbf{g}$, View of the binding groove from the top. Surface representation of the Fab with EBNA $1_{A A 386-402}$ in stick representation. $\mathbf{h}$, Cartoon and stick representation outlining close interactions. Major $\mathrm{H}$-bond forming residues are represented as sticks. H-bonds < $3.2 \AA$ are represented as black dashed lines. i, Magnification of peptide in the hydrophobic cage, $\mathbf{j}$, magnification of region around Arg396 to emphasize polar contacts of $\mathrm{HC}$ residues with Arg396 and Arg397. k,l, Bio-layer interferometry measurement of MS39p2w174 (red) and germline (blue) affinity to EBNA1 full-length protein. $k, K_{D}$ in $M$, mean $K_{D} \pm S D$, calculated from four serial dilutions from one representative experiment out of three independent experiments, $* \star P=0.0043$, unpaired twotailed Student's t test. I, association and dissociation curves corresponding to (k). 

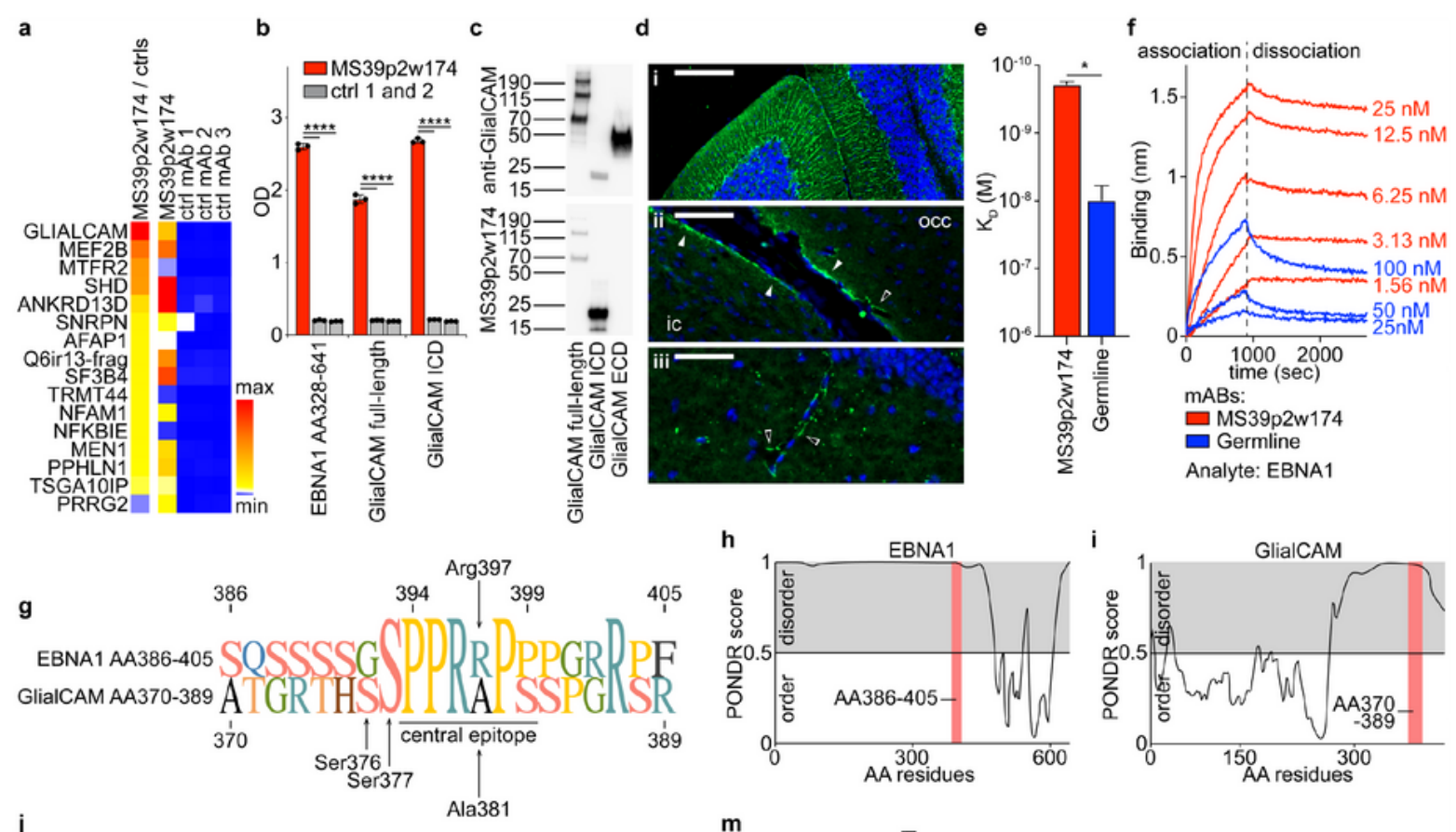

j

m
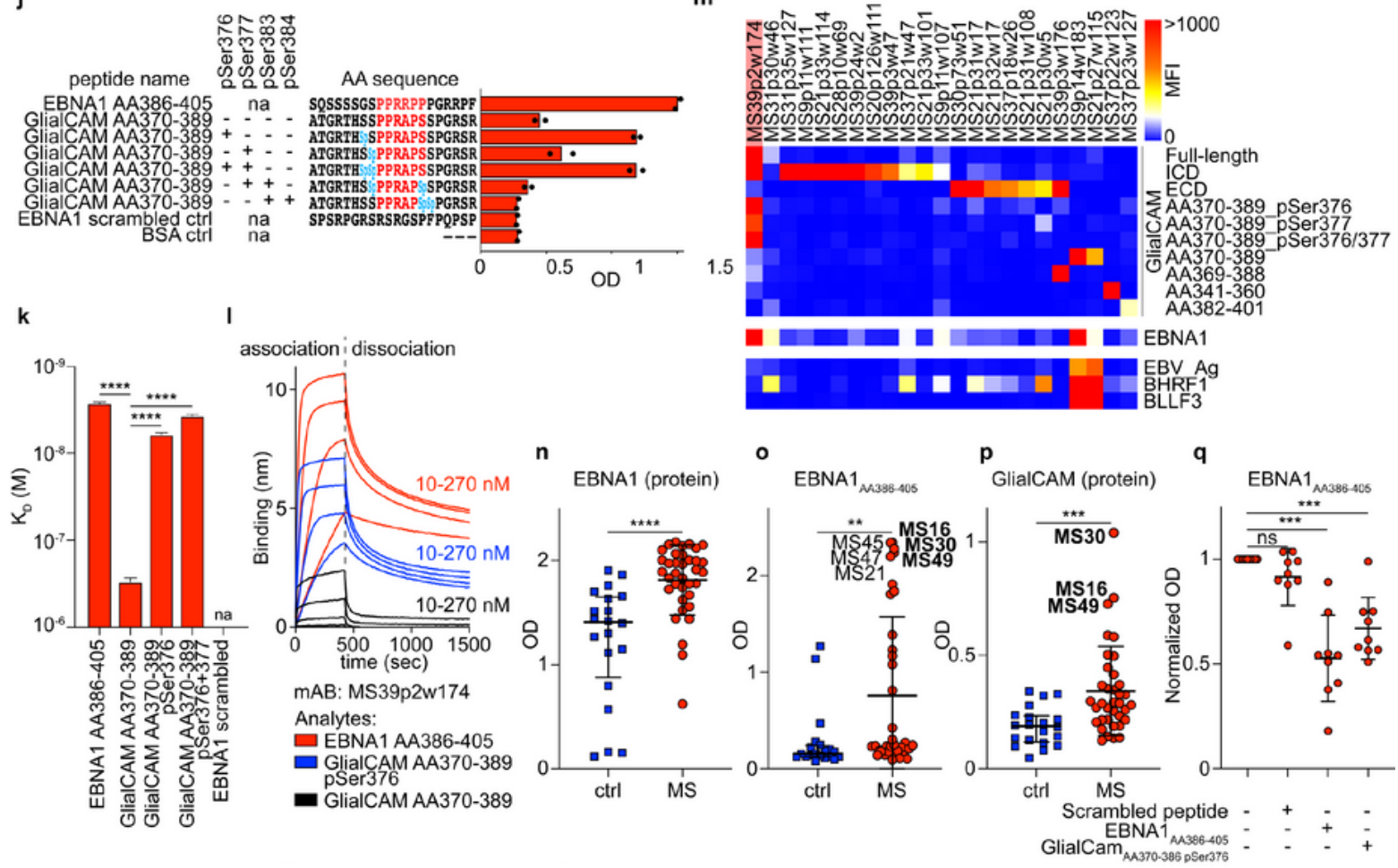

\section{Figure 3}

Molecular mimicry between EBNA1 and GlialCAM. a, Protein microarray data showing top 16 results of HuProt array for MS39p2w174, compared to 3 control mAbs, sorted from top to bottom by the ratio of MS39p2w174 / average of controls reactivity (left column, min: 89, max: 911). Raw counts are shown in the four columns on the right (min: 1, max: 36450). b, ELISA showing binding of MS39p2w174 and two control mAbs to recombinant proteins EBNA1 ${ }_{\text {AA328-641 }}$ as well as GlialCAM full-length (AA34-416) and 
ICD (AA262-416). Means \pm SD of $n=3$ independent experiments are shown, each carried out in triplicates. c, Western blot analysis of recombinant GlialCAM (full-length, ICD, and ECD), stained on separate blots with commercial anti-GlialCAM antibody (top panel) and MS39p2w174 (bottom panel), molecular weight marker in kDa. d, Fluorescent immunohistochemistry of mouse brain regions, stained with MS39p2w174, (i) cerebellum, scale bar: $150 \mu \mathrm{m}$, (ii) inferior colliculus (ic) and occipital cortex (occ) with perivascular glia (unfilled arrows) and glia limitans staining (filled arrows), scale bar: $60 \mu \mathrm{m}$, (iii) dentate gyrus with perivascular staining (unfilled arrows), scale bar: $40 \mu \mathrm{m}$. e,f, Bio-layer interferometry measurement of MS39p2w174 (red) and germline (blue) affinity to EBNA1 full-length protein. $k, K_{D}$ in $M$, mean $K_{D} \pm S D$, calculated from three serial dilutions from one representative experiment out of three independent experiments, ${ }^{\star} P=0.0124$, unpaired two-tailed Student's t test, $\mathbf{f}$, association and dissociation curves corresponding to (e). g, Alignment of AA sequences of EBNA1 ${ }_{A A 386-405}$ and GlialCAM ${ }_{\text {AA370-389 }}$ and pointing out the central epitope region. $\mathbf{h}, \mathbf{i}$, Prediction of disorder with PONDR for $\mathbf{h}$, EBNA1 and $\mathbf{i}$, GlialCAM. High scores indicate disorder, red areas: epitope regions. j, ELISA data showing binding of MS39p2w174 to EBNA1 ${ }_{A A 386-405}$ and GlialCAM ${ }_{\text {AA370-389 }}$ non-phosphorylated and phosphorylated at the indicated serine residues, means from $n=2$ independent experiments are shown, representative for 5 experiments, each carried out in duplicates. pSer / Sp: phosphorylated serine. k,l, Bio-layer interferometry measurement of MS39p2w174 affinity to GlialCAM 20mer peptides. $K_{D}$ in $M$, mean $K_{D} \pm S D$, calculated from four serial dilutions from one representative experiment out of three independent experiments, $\star \star \star \star ~ P$ $<0.0001$, unpaired two-tailed ANOVA, Tukey corrected for multiple comparisons. pSer: phosphorylated serine residues. I, association and dissociation curves corresponding to (k). $\mathbf{m}$, Protein microarray data showing mAb reactivities (MFI) to GlialCAM proteins, peptides, and phosphorylated peptides, as well as cross-reactivities to EBNA1 and other EBV proteins. Data from one experiment carried out in 8 technical replicates. ICD: intracellular domain, ECD: extracellular domain, pSer: phosphorylated serine residues. n-p, ELISA data showing human plasma reactivities against $\mathbf{n}$, EBNA1 protein AA328-641, ${ }^{\star \star \star *} P<0.0001$, unpaired two-tailed Mann-Whitney test, $\mathbf{0}, \mathrm{EBNA}_{\mathrm{AA} 386-405}, * * P<0.0044$, unpaired two-tailed MannWhitney test, and $\mathbf{p}$, GlialCAM protein, $* \star \star P<0.0002$, unpaired two-tailed Mann-Whitney test, means \pm SD from $n=20$ control individuals and $n=36$ MS patient samples. q, ELISA measurements of plasma antibody reactivity against $E B N A 1_{A A 386-405}$, without interference as well as blocked with scrambled peptide control,

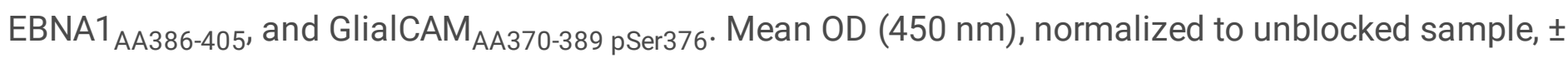
SD of $n=9$ patient samples, each measured in quadruplicates. e,k,n-p, $P$ values according to unpaired twosided Student's t-test, $\mathbf{q}$, one-way ANOVA, Dunnett corrected for multiple comparisons. ${ }^{*} P<0.05$, ${ }^{\star \star} P<$ $0.01, * \star \star P<10^{-3}, \star \star \star \star * P<10^{-4}$. 

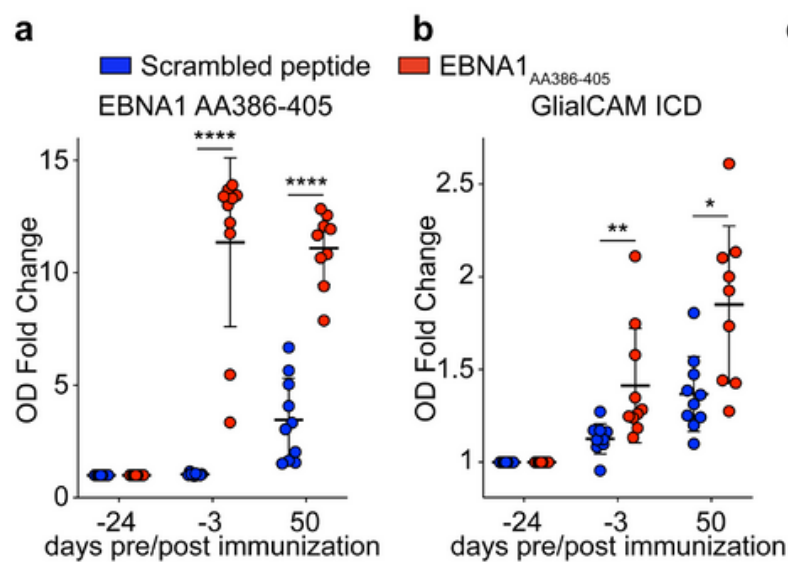

C

$\square$ Scrambled peptide $\square$ EBNA $_{\text {AA386-405 }}$

e
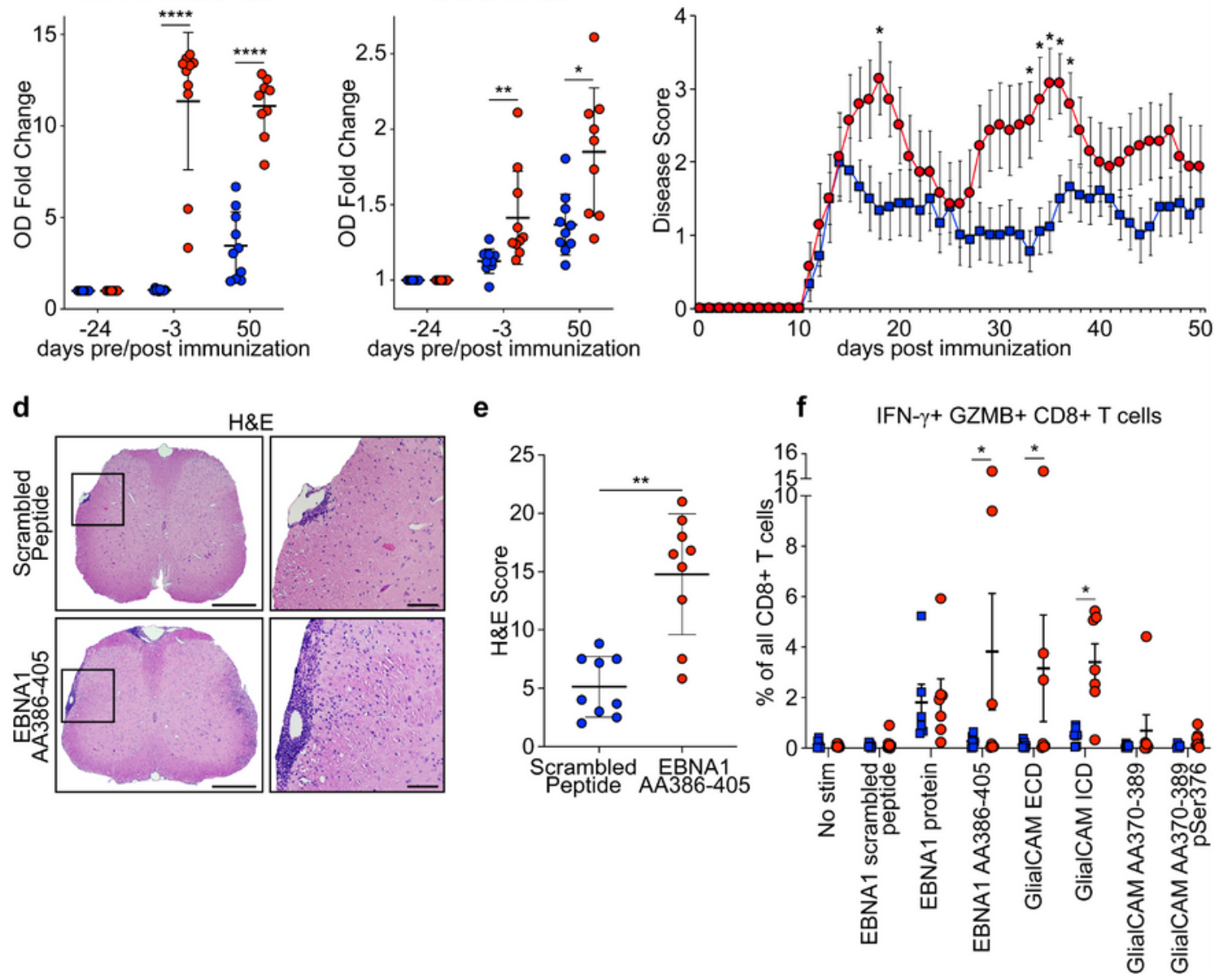

\section{Figure 4}

Anti-EBNA1 ${ }_{\mathrm{AA386-405}}$ immunization exacerbates autoimmune-mediated demyelination in vivo. a,b, ELISA data showing mouse plasma IgG response at the indicated timepoints pre and post EAE induction, for scrambled peptide immunized mice (blue, $n=10$ ) and EBNA1 ${ }_{\text {AA386-405 }}$ immunized mice (red, $n=10$ ) against

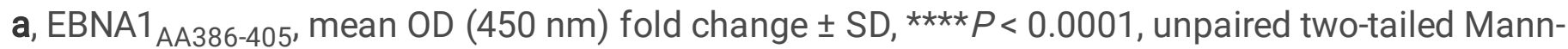
Whitney test, Holm-Sidak corrected for multiple comparisons, and b, GliaICAM ICD, mean OD (450 nm) 
fold change $\pm \mathrm{SD},{ }^{\star *} P=0.0022,{ }^{*} P=0.0152$, unpaired two-tailed Mann-Whitney test, Holm-Sidak corrected for multiple comparisons. Means \pm SD, representative of three independent experiments, each carried out as triplicate measurements. C, EAE scores of mice immunized with scrambled peptide (blue, $n=9$ ) and EBNA1 ${ }_{\mathrm{AA} 386-405}$ (red, $\left.n=7\right), 3$ weeks prior and on the day of EAE immunization (day 0), means of clinical scores \pm SEM, ${ }^{*} P<0.05$ unpaired two-tailed Mann Whitney test. d,e, Spinal cord histology, d, representative H\&E-stained spinal cords from scrambled peptide group (top panel) and EBNA1 ${ }_{A A 386-405}$ group (bottom panel). Scale bars left images: $200 \mu \mathrm{m}$, right images: $50 \mu \mathrm{m}$. e, Statistical evaluation of H\&E score, means of at least 4 coronal spinal cord sections per mouse and means \pm SD for each group $(n=9), * \star P=0.0012$, unpaired two-tailed Mann-Whitney test. f, Flow cytometry data of PBMC from healthy control individuals ( $n=6$, blue) and MS patients ( $n=7$, red), showing percent of IFN- -+ GZMB+ CD $8+$ T cells in all CD $8+T$ cells. Means \pm SEM are shown for the respective groups. Significance levels were assessed by two-way ANOVA, followed by FDR calculation using the two-stage step-up method of Benjamini, Krieger and Yekutieli, * FDR $<0.1$.

\section{Supplementary Files}

This is a list of supplementary files associated with this preprint. Click to download.

- ExtDataFigure1v14NatRev.tif.png

- ExtDataFigure2v12NatRev.tif.png

- ExtDataFigure3v9NatRev.tif.png

- ExtDataFigure4v12NatRev.tif.png

- ExtDataFigure5v11NatRev.tif.png

- ExtDataFigure6v6NatRev.tif.png

- ExtDataFigure7v12NatRev.tif.png

- ExtDataFigure8v16NatRev.tif.png

- ExtDataFigure9V2.tif.png

- ExtDataFigure10v3NatRev.tif.png

- 9NatRev2SupplInfoV3.pdf

- ExtDataTable1V4PatientCollective.pdf

- ExtendedDataTable2v2NatPhageDisplay.pdf 